\title{
Synergistic cardioprotective effects of Danshensu and hydroxysafflor yellow A against myocardial ischemia-reperfusion injury are mediated through the Akt/Nrf2/HO-1 pathway
}

\author{
TIANXIN HU, GUO WEI, MIAOMIAO XI, JIAJIA YAN, XIAOXIAO WU, \\ YANHUA WANG, YANRONG ZHU, CHAO WANG and AIDONG WEN
}

Department of Pharmacy, Xijing Hospital, The Fourth Military Medical University, Xi'an, Shaanxi 710032, P.R. China

Received July 12, 2015; Accepted April 27, 2016

DOI: $10.3892 /$ ijmm.2016.2584

\begin{abstract}
In clinical practice, the traditional Chinese medicinal herbs, Radix Salvia Miltiorrhiza and Carthamus tinctorius L., are usually prescribed in combination due to their significant cardioprotective effects. However, the mechanisms responsible for these combined effects remain unknown. Thus, in this study, we investigated the mechanisms responsible for the combined effects of Danshensu (DSS) and hydroxysafflor yellow A (HSYA) by establishing a rat model of myocardial ischemia/reperfusion (MI/R), as well as a model of hypoxia/reoxygenation (H/R) using H9c2 cells. The combination index (CI) was calculated using the median-effect method. DSS and HSYA in combination led to a CI value of $<1$ as regards infarct size in vivo and cell viability in vitro. The rats with $\mathrm{MI} / \mathrm{R}$ injury that were treated with DSS and/or HSYA were found to have significantly lower levels of creatine kinase-MB (CK-MB) and cardiac troponin I (cTnI) and malondialdehyde (MDA), and a lower expressoin of 8-hydroxydeoxyguanosine (8-OHdG), and markedly enhanced superoxide dismutase (SOD) activity. Our in vitro experiments revealed that the cells treated with DSS and/or HSYA had a reduced lactate dehydrogenase (LDH)
\end{abstract}

Correspondence to: Dr Aidong Wen, Department of Pharmacy, Xijing Hospital, The Fourth Military Medical University, 15 Changle West Road, Xi'an, Shaanxi 710032, P.R. China

E-mail: aidongwen2014@163.com

Abbreviations: $\mathrm{MI} / \mathrm{R}$, myocardial ischemia-reperfusion; Nrf2, nuclear factor erythroid 2-related factor 2; CI, combination index; DSS, Danshensu; SOD, superoxide dismutase; HSYA, hydroxysafflor yellow A; ROS, reactive oxygen species; Akt, protein kinase B; HO-1, heme oxygenase-1; H/R, hypoxia/reoxygenation; CK-MB, creatine kinase-MB; cTnI, cardiac troponin I; MDA, malondialdehyde; LDH, lactate dehydrogenase; TUNEL, terminal deoxynucleotidyl transferase-mediated dUTP nick-end labeling; 8-OHdG, 8-hydroxydeoxyguanosine

Key words: myocardial ischemia/reperfusion, Danshensu, hydroxysafflor yellow A, synergy, anti-apoptosis, antioxidant, protein kinase $\mathrm{B} /$ nuclear factor erythroid 2-related factor $2 /$ heme oxygenase-1 activity and a decreased percentage of cell apoptosis (increased Bcl-2/Bax ratio, decreased expression of cleaved caspase3). DSS and HSYA increased the expression of heme oxygenase-1 (HO-1), the phosphorylation of Akt and the translocation of nuclear factor erythroid 2-related factor 2 ( $\mathrm{Nrf} 2)$. Furthermore, the Akt inhibitor, LY294002, partially hampered the expression of Nrf2 and HO-1. The HO-1 inhibitor, zinc protoporphyrin IX (ZnPP-IX), did not decrease the expression of p-Akt and Nrf2, although it abolished the anti-apoptotic and antioxidant effects of DSS and HSYA. The findings of our study thus demonstrate that DSS and HSYA confer synergistic cardioprotective effects through the Akt/Nrf2/HO-1 signaling pathway, to certain extent, by enhancing the antioxidant defense system and exerting anti-apoptotic effects.

\section{Introduction}

Myocardial infarction is a leading cause of mortality and morbidity in developed countries. Although prompt reperfusion therapy has been shown to reduce the infarct size and improve left ventricular function in ST-elevation myocardial infarction, reperfusion itself may cause lethal tissue injury and a series of cellular events termed myocardial ischemia-reperfusion (MI/R) injury (1-4).

The molecular mechanisms of reperfusion injury appear to be multifactorial with various consequences on cellular function (1). It has been previously noted that apoptosis occurs shortly after myocardial infarction and it is markedly enhanced during reperfusion $(5,6)$. The production of reactive oxygen species (ROS) in the ischemic region and the surrounding myocardium is also generated, which directly triggers cell death, including apoptosis. Inflammatory responses, disruptions of energy metabolism and calcium overload have also been proposed to underlie the pathology of MI/R injury (7). Thus, a single therapeutic agent may be incapable of hitting multiple targets to achieve therapeutic efficacy.

Comprehensive investigations have been focusing on combination drugs in order to optimize or amplify the therapeutic effects $(8,9)$. In traditional Chinese medicine (TCM), a number of herbs are paired together in order to attenuate toxicity, as well as to enhance the therapeutic effects (10). Radix Salvia miltiorrhiza (S. miltiorrhiza) and Carthamus tinc- 
torius L. (C. tinctorius; also known as Flos Carthami) are usually used as a combination herbal formulation, known as Danhong injection, which can relieve the symptoms of angina pectoris, attenuate myocardial ischemia and promotes atherosclerotic plaque regression (11). Due to their complex constituents, studies have mainly focused on their combination effects. However, previous studies have confirmed that Danshensu (DSS) and hydroxysafflor yellow A (HSYA) are the main active ingredients of Radix Salvia miltiorrhiza and Flos Carthami, respectively $(12,13)$. Hence, evaluating the combination effects of these two active compounds may be of importance in understanding the rationale for the combined use of the two herbs in TCM.

There is evidence to indicate that S. miltiorrhiza and C.tinctorius inhibit cellular apoptosis and oxidative stress induced by MI/R (14,15). It would be of interest to investigate the mechanisms reponsible for the combined effects of DSS and HSYA. Thus, the present study aimed to evaluate the cardioprotective effects of combination therapy with DSS and HSYA in order to elucidate the mechanisms responsible for their combined antioxidant and anti-apoptotic effects in vivo and in vitro.

\section{Materials and methods}

Chemicals and drugs. DSS was purchased from the National Institute for the Control of Pharmaceutical and Biological Products (Beijing, China) as an amorphous powder. Its purity (>98\%) was determined using high-performance liquid chromatography (HPLC). The molecular formula of DSS is $\mathrm{C}_{9} \mathrm{H}_{10} \mathrm{O}_{5}$ and its molecular weight is 162.14 . HSYA was obtained from $C$. tinctorius as a yellow amorphous powder. Its purity (>99\%) was determined using HPLC. Being soluble in water, it has a molecular formula of $\mathrm{C}_{27} \mathrm{H}_{32} \mathrm{O}_{16}$ and a molecular weight of 611.1614 .

The creatine kinase-MB (CK-MB) and cardiac troponin I (cTnI) kits were purchased from Roche Molecular Biochemicals (Mannheim, Germany). The lactate dehydrogenase (LDH), superoxide dismutase (SOD) and malondialdehyde (MDA) kits were obtained from Jiancheng Bioengineering Institute (Nanjing, China).

Dulbecco's modified Eagle's medium (DMEM) and other cell culture supplies were purchased from Gibco-BRL (Grand Island, NY, USA). 3-(4,5-Dimethylthiazol-2-yl)-2,5diphenyltetrazolium bromide (MTT), zinc protoporphyrin IX [ZnPP-IX; a heme oxygenase-1 (HO-1) inhibitor], 2,3,5-triphenyltetrazolium chloride (TTC), Evans blue, and hematoxylin and eosin were the products of Sigma Chemical Co. (St. Louis, MO, USA). The phosphoinositide 3-kinase (PI3K) inhibitor, LY294002 (\#9901), and rabbit polyclonal antibodies specific for Bcl-2 (\#2870), Bax (\#2772), $\beta$-actin (\#4970), total protein kinase B (Akt; t-Akt, serine 473; \#9272), phosphorylated Akt (p-Akt; \#9271), cleaved caspase-3 (\#9661) and caspase-3 (\#9662) were obtained from Cell Signaling Technology (Beverly, MA, USA). Rabbit polyclonal antibodies specific for HO-1 (\# sc-10789), 8-hydroxydeoxyguanosine (8-OHdG; \#sc-66036) and nuclear factor erythroid 2-related factor 2 (Nrf2; \#sc-722) were purchased from Santa Cruz Biotechnology, Inc. (Santa Cruz, CA, USA). Rabbit polyclonal antibody specific for histone 3 (H3; \#ab4729) was purchased from Abcam (Cambridge, UK). All materials for sodium dodecyl sulfate-polyacrylamide gel electrophoresis (SDS-PAGE) were obtained from Bio-Rad Laboratories (Hercules, CA, USA).

\section{Experimental protocols}

In vivo experiments. In consideration of the failures which may occur when performing coronary ligation, such as no infarcts or death, 10 rats were randomly selected in each group so that a sufficient number of animals (at least 6 rats) was maintained. The concentrations of DSS and HSYA were $10,20,30,45$ and $60 \mathrm{mg} / \mathrm{kg}$, and 10, 20, 30, 50 and $70 \mathrm{mg} /$ $\mathrm{kg}$, respectively. They were combined at concentrations of 10 , $20,30,50$ and $70 \mathrm{mg} / \mathrm{kg}$ (at a ratio of 1:1 according to each $\mathrm{IC}_{50}$ value) to analyze the synergistic effects on myocardial infarct size. In the subsequent experiments, the rats were randomly divided into 5 groups as follows: i) the sham-operated group, ii) the MI/R group, iii) the MI/R + DSS (60 mg/ $\mathrm{kg}$ ) group, iv) the MI/R + HSYA (70 $\mathrm{mg} / \mathrm{kg}$ ) group, and v) the $\mathrm{MI} / \mathrm{R}+\mathrm{DSS}+\mathrm{HSYA}(\mathrm{DH} ; 35 \mathrm{mg} / \mathrm{kg} \mathrm{DSS}+35 \mathrm{mg} / \mathrm{kg}$ HSYA) group. All drugs were administered via tail vein injection at the time of reperfusion. The concentrations of DSS and HSYA were selected on the basis of the reported literature and our preliminary dose selection experiments $(15,16)$.

In vitro experiments. To further explore the mechanisms responsible for the combined effects of DSS and HSYA, H9c2 cardiomyocytes were used. The concentrations of DSS and HSYA were 1, 10, 35, 60 and $80 \mu \mathrm{M}$. DSS and HSYA at concentrations of $1,10,35,60$ and $80 \mu \mathrm{M}$ were used in combination (at a ratio of $1: 1$ according to each $\mathrm{IC}_{50}$ value) to analyze the synergistic effects on cell viability. In the subsequent experiments, the H9c2 cells were randomly divided into the following groups: i) the control (Con) group, ii) the hypoxia/reoxygenation $(\mathrm{H} / \mathrm{R})$ group, iii) the $\mathrm{H} / \mathrm{R}+\mathrm{DSS}(80 \mu \mathrm{M})$ group, iv) the $\mathrm{H} / \mathrm{R}+\mathrm{HSYA}(80 \mu \mathrm{M})$ group, v) the H/R + DH (40 $\mu \mathrm{M}$ DSS + $40 \mu \mathrm{M}$ HSYA) group, vi) the $\mathrm{H} / \mathrm{R}+\mathrm{DH}+\mathrm{ZnPP}-\mathrm{IX}(10 \mu \mathrm{M})$ group, and vii) the $\mathrm{H} / \mathrm{R}+\mathrm{DH}+\mathrm{LY} 294002(50 \mu \mathrm{M})$ group. The doses of each agent were selected according to the published literature and our preliminary experiments $(17,18)$.

Animals and the establishment of an animal model of $M I / R$ injury. Adult male Sprague-Dawley rats $(n=220$, approximately 2 months old, weighing $250 \pm 20$ g) were purchased from the Experimental Animal Research Center at the Fourth Military Medical University (Xi'an, China). The experimental protocols involving animals were performed in adherence with Institutional Animal Care and were approved by the Animal Care and Use Committee of the Fourth Military Medical University.

The rats were anesthetized with sodium pentobarbital (40 mg/kg intraperitoneally) and the tracheas were cannulated with a polyethylene-90 (PE-90) tube connected to a rodent ventilator with a tidal volume of $1.21 / \mathrm{kg}$ (75 breaths $/ \mathrm{min}$ ). A left thoracotomy was performed between the fourth and fifth ribs. The pericardium was removed and the left anterior descending (LAD) artery was visualized and ligated with a 6-0 Prolene suture, as previously described (19). The appearance of myocardial pallor was confirmed as ischemia. After 30 min of LAD ligation, the ligature was removed to allow for $180 \mathrm{~min}$ of reperfusion. In the sham-operated group, Prolene was drilled underneath the LAD, but not ligated. 
Assessment of myocardial infarct size. After the completion of $180 \mathrm{~min}$ of reperfusion, the myocardial infarct size was assessed by a double-staining technique using 2\% TTC and $3 \%$ Evans blue as previously described (20). Briefly, the LAD was re-ligated and $2 \mathrm{ml}$ of $3 \%$ Evans blue dye was retrogradely infused into the carotid artery to demarcate the area at risk (AAR; area not perfused with blue dye) from the area not at risk (stained with blue dye). After the dye was uniformly distributed, the hearts were rapidly excised and frozen at $-20^{\circ} \mathrm{C}$, and subsequently the heart tissue was cut into 5 transverse slices. The sections were incubated in $2 \%$ TTC solution in phosphate buffer ( $\mathrm{pH} \mathrm{7.4)}$ at $37^{\circ} \mathrm{C}$ in the dark for $15 \mathrm{~min}$ and then stored in $4 \%$ paraformaldehyde overnight to delineate the infarct size (IS; pale area). The IS and AAR were measured using Image-Pro Plus software (Media Cybernetics, Inc., Silver Spring, MD, USA) after capturing images. The myocardial infarct size was calculated as a percentage of the infarct size over the total AAR. The dose-effect curve and fraction versus combination index $(\mathrm{Fa}-\mathrm{CI})$ curve were analyzed using CompuSyn software (MIT, Cambridge, MA, USA).

Determination of $C K-M B$ and $c T n I$ release in serum. After being anesthetized, the rats were subjected to MI/R surgery. Following $3 \mathrm{~h}$ of reperfusion, blood samples were collected from the abdominal aorta of the rats using a $10 \mathrm{ml}$ syringe. The blood of the experimental rats was collected and serum was separated by centrifugation and then kept at $-20^{\circ} \mathrm{C}$. The levels of CK-MB, and cTnI were estimated using a commercially available enzyme-linked immunosorbent assay kit with a microplate reader (Thermo Fisher Scientific, Waltham, MA, USA) according to the manufacturer's instructions.

Assessment of markers for oxidative stress. The serum and the cell culture supernatant were used to assay the MDA content and SOD activity using a microplate reader (Multiskan GO; Thermo Fisher Scientific) according to the instructions provided by the manufacturer.

Immunohistochemical assay for the evaluation of 8-OHdG expression. The paraffin-embedded tissue samples were deparaffinaged in xylene and then dehydrated with ethanol. Subsequently, the samples were subjected to antigen retrieval and then incubated in $3 \% \mathrm{H}_{2} \mathrm{O}_{2}$ in $0.01 \mathrm{M}$ phosphate-buffered saline (PBS) and in 5\% bovine serum albumin (BSA) successively. The sections were incubated overnight at $4^{\circ} \mathrm{C}$ with a primary antibody anti-8-OHdG (1:100) and then incubated for $1 \mathrm{~h}$ with a secondary antibody (Boster Biological Technology, Wuhan, China). The reaction was visualized with a solution of diaminobenzidine (DAB) and counterstained with hematoxylin. For quantification, the number of $8-\mathrm{OHdG}$-stained positive cells was calculated using Image-Pro Plus 6.0 software (Media Cybernetics, Inc.).

Cell culture and $H / R$ injury. The H9c2 cardiomyocyte cell line was purchased from the Chinese Academy of Sciences Cell Bank (Shanghai, China) and maintained in DMEM supplemented with $10 \%(\mathrm{v} / \mathrm{v})$ fetal bovine serum at $37^{\circ} \mathrm{C}$ in a $\mathrm{CO}_{2}$ incubator. The medium was replaced every 2-3 days, and the cells were subcultured or subjected to experimental procedures at $80-90 \%$ confluence.
To mimic ischemic injury in vivo, the procedures for inducing $\mathrm{H} / \mathrm{R}$ injury were modified from a previously described method (21). Briefly, the cells were maintained in serum-free DMEM (glucose-free) instead of routine culture medium. Hypoxic conditions were established by equilibrating a humidified chamber containing the cells with $95 \% \mathrm{~N}_{2}$ and $5 \% \mathrm{CO}_{2}$ via a gas transfusion apparatus (Billups-Rothenberg, Del Mar, CA, USA). Following $4 \mathrm{~h}$ of incubation, the cells were transferred to normal conditions in a $\mathrm{CO}_{2}$ incubator and the medium was replaced with routine culture medium to achieve re-oxygenation. The drugs were administered at the beginning of re-oxygenation. Following $20 \mathrm{~h}$ of re-oxygenation, the culture medium was collected and stored at $-80^{\circ} \mathrm{C}$ until analysis. In the control group, the $\mathrm{H} 9 \mathrm{c} 2$ cells were cultured under normal conditions for $24 \mathrm{~h}$.

Analysis of cell viability and LDH activity. The cells in the exponential phase were seeded at $1 \times 10^{4}$ cells/well in 96-well plates. After being subjected to the different treatments, $20 \mu \mathrm{l}$ of MTT solution were added to the medium $(0.5 \mathrm{mg} / \mathrm{ml}$ final concentration in medium) and the cells were incubated for an additional $4 \mathrm{~h}$ at $37^{\circ} \mathrm{C}$. The supernatants were removed and the formazan crystals were dissolved in $150 \mu \mathrm{l}$ DMSO. The absorbance was read at $490 \mathrm{~nm}$ using a microplate reader (Multiskan GO; Thermo Fisher Scientific). A reduction in optical density reduction was considered to indicate a decrease in cell viability. The cells in the control group were considered $100 \%$ viable. The results were also assessed using Compusyn software to calculate the CI value.

In order to confirm the degree of cardiomyocyte injury in $\mathrm{H} / \mathrm{R}$, the release of $\mathrm{LDH}$ was measured. After being subjected to the different treatments, the cell culture supernatants were collected to assay LDH activity immediately according to the manufacturer's instructions using a microplate reader (Multiskan GO; Thermo Fisher Scientific) at $450 \mathrm{~nm}$.

Protein extraction and western blot analysis. The cardiomyocytes were resuspended in radioimmunoprecipitation assay (RIPA) lysis buffer (Beyotime Institute of Biotechnology, Jiangsu, China) on ice for $30 \mathrm{~min}$. They were then centifuged at $4^{\circ} \mathrm{C}$ for $20 \mathrm{~min}$ at $10,000 \mathrm{rpm}$ to separate the supernatant before being stored at $-80^{\circ} \mathrm{C}$. The nuclear proteins were extracted separately from cultured myocytes using NE-PER nuclear and cytoplasmic extraction reagents according to the manufacturer's instructions (ThermoFisherScientific). A Mitochondria/Cytosol Fractionation kit (BioVision, San Francisco, CA, USA) was used to prepare mitochondrial protein. Protein concentrations were measured using the Bradford method with the Bio-Rad protein assay kit (Bio-Rad Laboratories). Denatured protein was separated by SDS-PAGE and then electrotransferred onto polyvinylidene difluoride (PVDF) membranes (Millipore, Billerica, MA, USA). After being blocked with $5 \%(\mathrm{w} / \mathrm{v})$ non-fat milk at $37^{\circ} \mathrm{C}$ for $30 \mathrm{~min}$, the membranes were incubated overnight at $4^{\circ} \mathrm{C}$ with primary antibodies including $\mathrm{p}$-Akt, t-Akt, Bcl-2, Bax, cleaved caspase-3 and caspase-3, HO-1, Nrf2, $\beta$-actin and $\mathrm{H} 3$ (1:1,000), respectively. After washing in TBST 3 times, the membranes were incubated at room temperature for $1 \mathrm{~h}$ with secondary antibody diluted in TBST. The labeled protein bands were detected using chemiluminescent reagents and were exposed to film. The band intensity was determined 
using an image analyzer (Quantity One System; Bio-Rad, Richmond, CA, USA).

Detection of apoptotic cell death. Cell apoptosis was determined by terminal deoxy-nucleotidyl transferase-mediated dUTP nick-end labeling (TUNEL) assay, as previously described (22). The H9c2 cells grown on a 6-mm plate were fixed with $4 \%$ paraformaldehyde solution for $30 \mathrm{~min}$ at room temperature. The cells were then treated with permeation solution. Subsequently, the samples were incubated with TUNEL reagent. The cells were also stained with $1 \mu \mathrm{g} / \mathrm{ml}$ 4',6-diamidino-2-phenylindole (DAPI) for $30 \mathrm{~min}$ to detect cell nuclei (blue). The number of TUNEL-positive cells was presented as a percentage of the total cardiomyocytes and was evaluated at $\mathrm{x} 400$ magnification.

Determination of combined effects. The manner in which DSS and HSYA act with regard to myocardial infarct size and cell viability was determined by a median-effect method proposed by Chou (23). Synergism or antagonism was determined with CI values. CompuSyn software (MIT) was used to determine the $\mathrm{CI}$ value. The $\mathrm{CI}$ was plotted as the fractional inhibition $(\mathrm{Fa})$ by computer simulation from 0.10 to 0.95 . In this analysis, the combined effect at the $50 \%$ fractional inhibition $\left(\mathrm{CI}_{50}\right)$ was reported as synergistic, antagonistic or additive when the $\mathrm{CI}_{50}$ value was $<1,>1$ and equal to 1 , respectively.

Statistical analysis. All values are expressed as the means \pm standard deviation (SD). Statistical analysis was performed using one-way analysis of variance (ANOVA) followed by a least significant difference (LSD) test for multiple comparisons, using SPSS 19.0 software for Windows (SPSS Inc., Chicago, IL, USA). A value of $\mathrm{P}<0.05$ was considered to indicate a statistically significant difference.

\section{Results}

DSS and HSYA synergistically alleviate myocardial injury in rats with MI/R injury. Infarct size and the release of $\mathrm{cTnI}$, and CK-MB in serum were measured to determine the mechanisms through which DSS and HSYA influence MI/R injury. The myocardial infarct size was significantly reduced in the groups treated with the compounds compared with that in the MI/R group (Fig. 1A and B). Combined treatment (DH) enhanced this effect to a greater extent than treatment with either DSS or HSYA alone. In order to determine whether DSS and HSYA have a synergistic effect on reducing the infarct size, the doseeffect curves of the single or combined treatment were analyzed by the median-effect method. We found that DSS, HSYA and combination therapy (Fig. 1C-1) at concentrations of 10-60, $10-70$ and $10-70 \mathrm{mg} / \mathrm{kg}$ yielded $\mathrm{CI}_{50}$ values $<1$ (Fig. 1C-2), indicating synergistic effects between the agents. As these effects occurred in a dose-dependent manner, the highest doses were selected for use in subsequent experiments. The numbers of rats that failed the coronary ligation procedure in each group shown in Fig. 1 are listed in Table I.

As markers of cardiomyocyte injury, the serum levels of CK-MB and cTnI were $104.68 \pm 9.93$ and $8.47 \pm 0.80 \mathrm{ng} / \mathrm{ml}$ in the sham-operated group and were significantly increased in the $\mathrm{MI} / \mathrm{R}$ group to $220.78 \pm 10.63$ and $21.52 \pm 2.79 \mathrm{ng} / \mathrm{ml}$, respectively.
Table I. Number of rats that failed the coronary ligation procedure in each group shown in Fig. 1.

\begin{tabular}{lclc}
\hline Group & No. & \multicolumn{1}{c}{ Group } & No. \\
\hline Sham & 1 & MI/R + HSYA30 & 1 \\
MI/R & 3 & MI/R + HSYA50 & 1 \\
MI/R + DSS10 & 2 & MI/R + HSYA70 & 3 \\
MI/R + DSS20 & 3 & MI/R + DH10 & 1 \\
MI/R + DSS30 & 2 & MI/R + DH20 & 2 \\
MI/R + DSS45 & 3 & MI/R + DH30 & 6 \\
MI/R + DSS60 & 2 & MI $/ R+D H 50$ & 2 \\
MI/R + HSYA10 & 1 & MI $/ R+D H 70$ & 1 \\
MI/R + HSYA20 & 2 & & \\
\hline
\end{tabular}

MI/R, myocardial ischemia-reperfusion; HSYA, hydroxysafflor yellow A; DSS, Danshensu; DH, HYSA + DSS.

Table II. Number of rats that failed the coronary ligation procedure in each group shown in Fig. 2.

Group Sham MI/R MI/R + DSS MI/R + HSYA MI/R + DH

\begin{tabular}{llllll}
\hline No. & 1 & 3 & 3 & 1 & 2 \\
\hline
\end{tabular}

MI/R, myocardial ischemia-reperfusion; HSYA, hydroxysafflor yellow A; DSS, danshensu; DH, HYSA + DSS.

Following treatment with $60 \mathrm{mg} / \mathrm{kg}$ DSS or $70 \mathrm{mg} / \mathrm{kg}$ HSYA, the CK-MB and cTnI levels were significantly decreased $(\mathrm{P}<0.01$ and $\mathrm{P}<0.05 \mathrm{vs}$. MI $/ \mathrm{R})$. The $\mathrm{DH}$ regimen $(35+35 \mathrm{mg} / \mathrm{kg})$ further enhanced these effects compared to treatment with each agent alone (Fig. 2). The numbers of rats that failed the coronary ligation procedure in each group shown in Fig. 2 are listed in Table II.

DSS and HSYA exert antioxidant effect on rats with $M I / R$ injury. The rats in the MI/R group exhibited a significant increase in the level of MDA to $17.01 \pm 3.58 \mathrm{nmol} / \mathrm{ml}(\mathrm{P}<0.01$ vs. sham-operated group) and a significant decrease in SOD activity to $69.66 \pm 16.32 \mathrm{U} / \mathrm{ml}$ ( $\mathrm{P}<0.01$ vs. sham-operated group). The administration of DSS and HSYA in combination to the rats with $\mathrm{MI} / \mathrm{R}$ injury exerted more significant cardioprotective effects $(\mathrm{P}<0.01 \mathrm{vs.} \mathrm{MI} / \mathrm{R})$ than the administration of each agent alone ( $\mathrm{P}<0.05 \mathrm{vs} . \mathrm{MI} / \mathrm{R})$, alleviating the parameters of oxidative stress (Table III). 8-OHdG is regarded as a hallmark of oxidative DNA damage (24); thus, nuclear oxidative stress was assessed using $8-\mathrm{OHdG}$ immunohistochemical staining, which was significantly increased $(\mathrm{P}<0.01$ vs. shamoperated group) in the MI/R group, while it was significantly reduced $(\mathrm{P}<0.01 \mathrm{vs}$. MI $/ \mathrm{R})$ in the combined treatment group. The protocol used in the DH group further potentiated the cardioprotective effects compared with those observed in the individual treatment groups (Fig. 3). Additionally, HSYA exerted a more potent antioxidant effect than DSS. The number of rats that failed the coronary ligation procedure in each group shown Fig. 3 are listed in Table IV. 


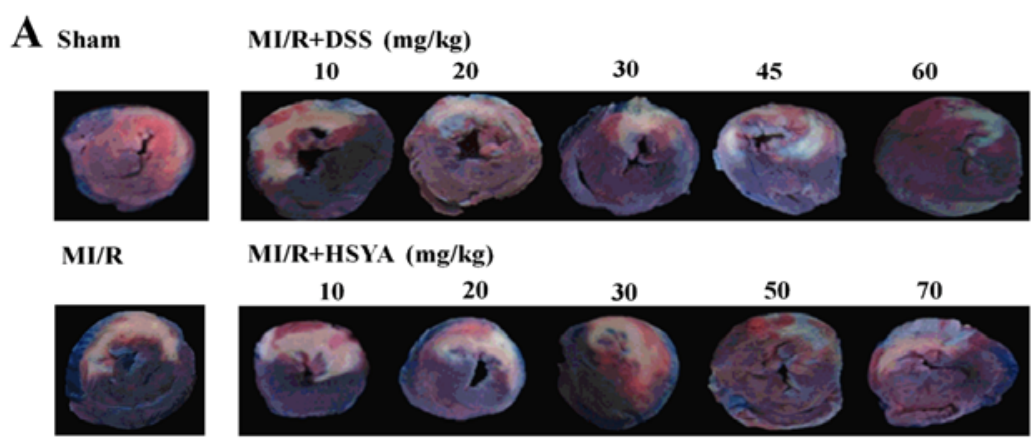

$\mathrm{MI} / \mathrm{R}+\mathrm{DH}(\mathrm{mg} / \mathrm{kg})$

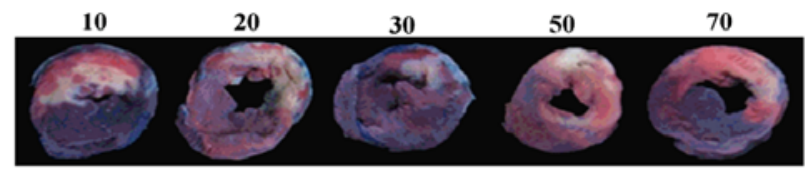

B

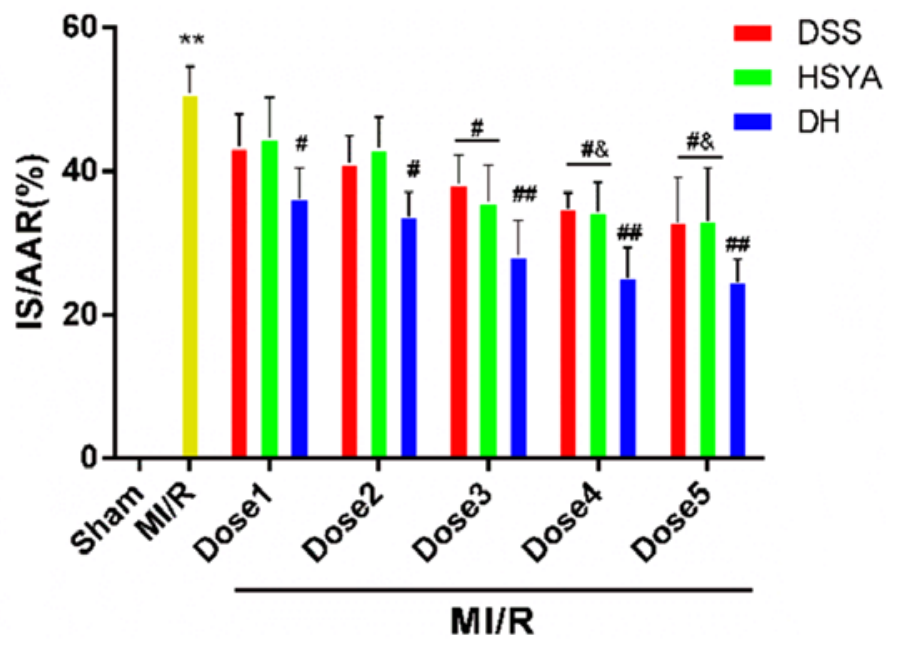

C-1

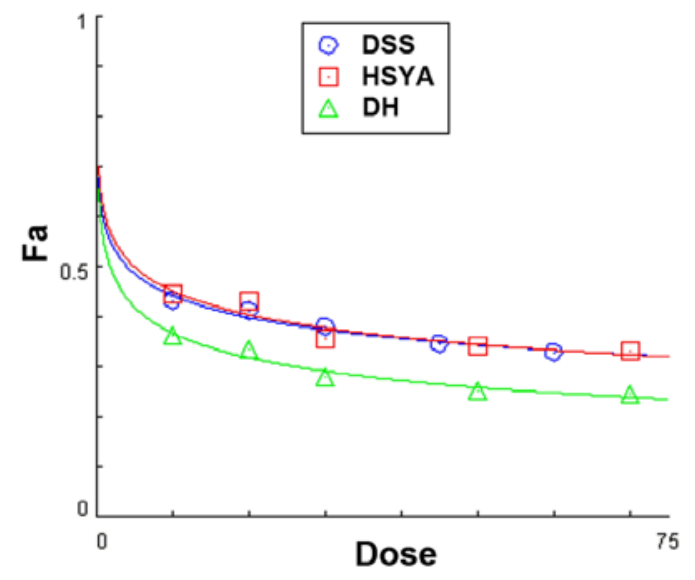

C-2

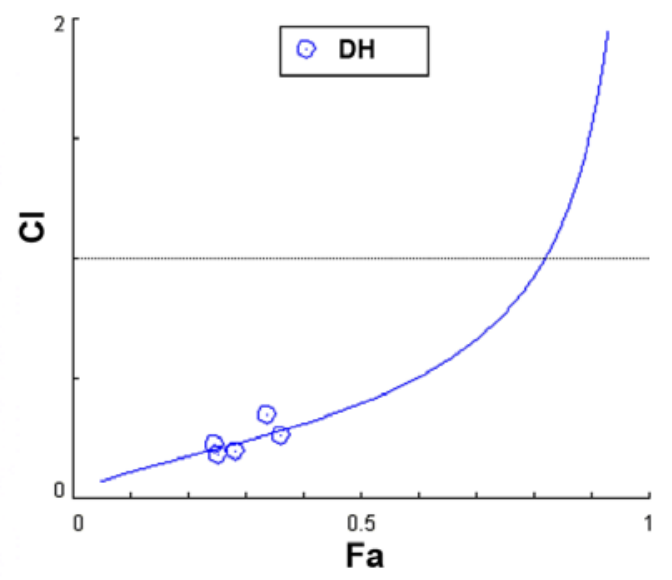

Figure 1. Danshensu (DSS) and hydroxysafflor yellow A (HSYA) administered individually and in combination (DH) reduce myocardial infarct size in rats subjected to myocardial ischemia-reperfusion (MI/R) injury, and in combination exert a synergistic effect. (A) A double-staining technique using TTC and Evans blue assessed the effect of DSS and HSYA, individually and in combination at different doses on infarct size and myocardial risk area. (B) Myocardial infarct size expressed as a percentage of the area at risk (IS/AAR\%). Dose 1 indicates DSS (10 mg/kg), HSYA (10 mg/kg), DH (10 mg/kg); Dose 2 indicates DSS (20 mg/ $\mathrm{kg})$, HSYA (20 mg/kg), DH (20 mg/kg); Dose 3 indicates DSS (30 mg/kg), HSYA (30 mg/kg), DH (30 mg/kg); Dose 4 indicates DSS (45 mg/kg), HSYA (50 mg/ $\mathrm{kg})$, DH (50 mg/kg); Dose 5 indicates DSS (60 mg/kg), HSYA (70 mg/kg), DH (70 mg/kg). (C-1) The dose-effect curves of the single or combined drug treatment. (C-2) The fraction versus combination index (Fa-CI) curve of DSS and HSYA in combination reveals that they exert a synergistic effect $\left(\mathrm{CI}_{50}<1\right)$ as reflected by the median-effect method. The dashed line at $\mathrm{CI}=1$ represents the additive effect. Values are expressed as the means $\pm \mathrm{SD}\left(\mathrm{n}=7-9\right.$ rats in each group). ${ }^{* *} \mathrm{P}<0.01 \mathrm{vs}$. sham-operated (sham) group; ${ }^{\# \#} \mathrm{P}<0.01$ and ${ }^{\#} \mathrm{P}<0.05$ vs. MI/R group; ${ }^{\circledR} \mathrm{P}<0.05$ vs. MI/R + DSS or MI/R + HSYA group.
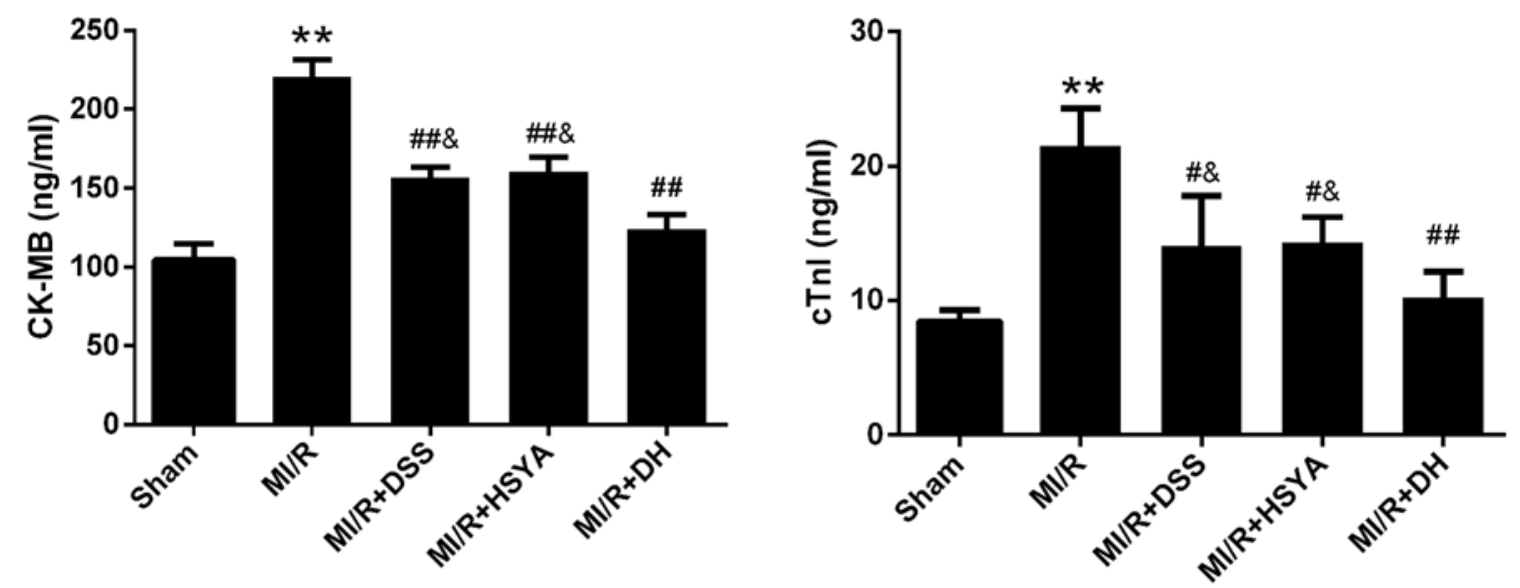

Figure 2. Levels of creatine kinase-MB (CK-MB) and cardiac troponin I (cTnI) in the serum of rats in the sham-operated (sham), myocardial ischemia-reperfusion (MI/R), MI/R + Danshensu (DSS; $60 \mathrm{mg} / \mathrm{kg}$ ), MI/R + hydroxysafflor yellow A (HSYA; $70 \mathrm{mg} / \mathrm{kg}$ ) and MI/R + DSS + HSYA (35+35 mg/kg) groups. Values are expressed as the means $\pm \mathrm{SD}\left(\mathrm{n}=7-9\right.$ rats in each group). ${ }^{* *} \mathrm{P}<0.01$ vs. sham group; ${ }^{\# \#} \mathrm{P}<0.01$ and ${ }^{\#} \mathrm{P}<0.05$ vs. MI/R group; ${ }^{\text {}} \mathrm{P}<0.05$ vs. MI/R $+\mathrm{DH}$ group. 

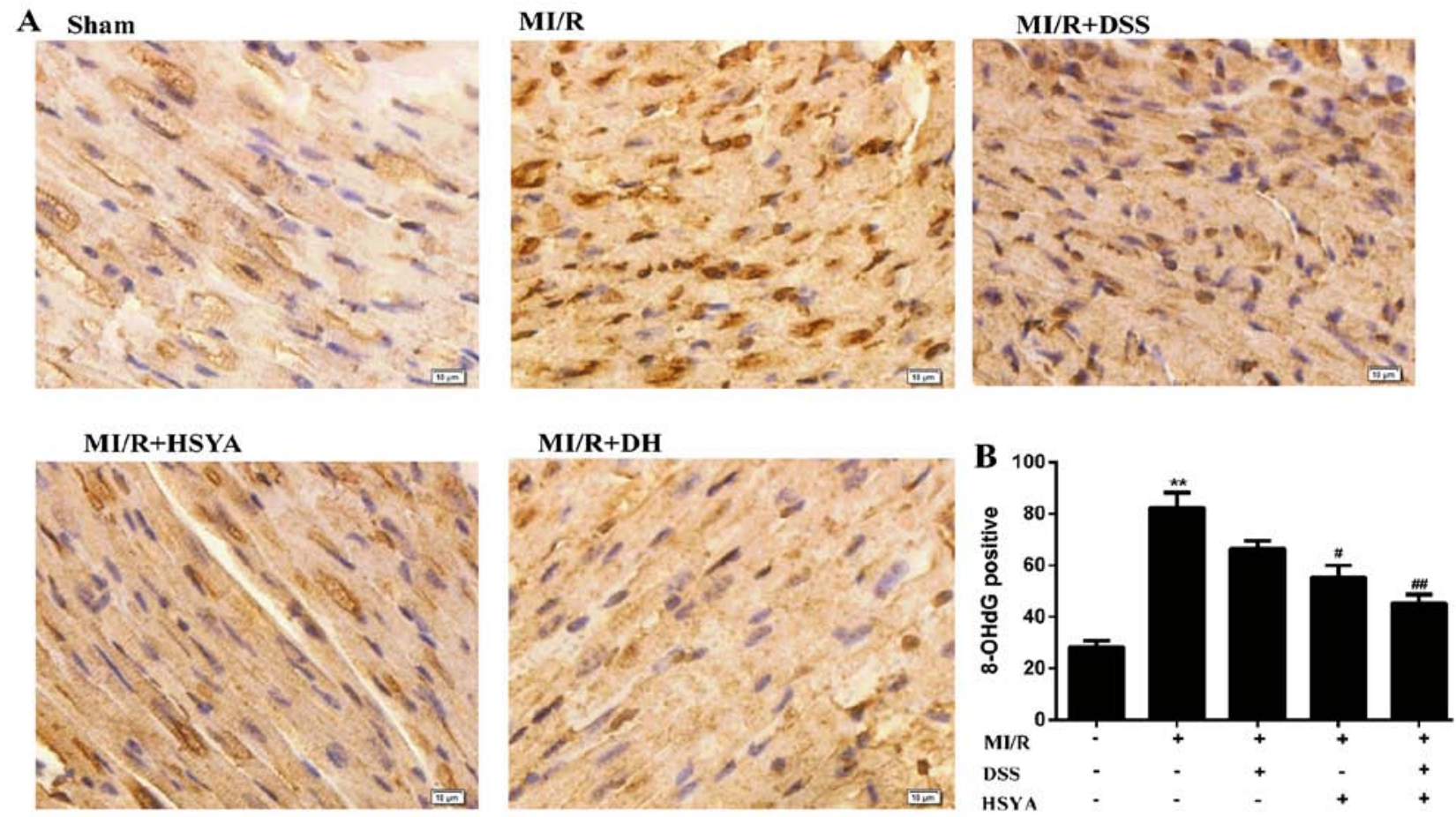

Figure 3. Effects of Danshensu (DSS) and hydroxysafflor yellow A (HSYA) administered individually or in combination evaluated by 8-hydroxydeoxyguanosine (8-OHdG) immunohistochemical staining in the sham-operated (sham), myocardial ischemia-reperfusion (MI/R), MI/R + DSS (60 mg/kg), MI $/ \mathrm{R}+$ HSYA $(70 \mathrm{mg} / \mathrm{kg}$ ) and MI/R + DSS + HSYA $(35+35 \mathrm{mg} / \mathrm{kg}$ ) groups. (A) Representative images of 8-OHdG staining. (B) The quantitative bar graph was generated from the number of 8-OHdG-positive cells in the myocardium (positive nuclei brown and negative nuclei blue). Values are expressed as the means $\pm \mathrm{SD}$; scale bar, $20 \mu \mathrm{m}$ ( $\mathrm{n}=7-9$ rats in each group). ${ }^{* *} \mathrm{P}<0.01$ vs. sham group; ${ }^{\#} \mathrm{P}<0.05$ and ${ }^{\# \#} \mathrm{P}<0.01 \mathrm{vs}$. MI/R group.

Table III. Levels of malondialdehyde (MDA) and superoxide dismutase (SOD) activity in the serum of rats.

\begin{tabular}{lcc}
\hline Treatment & $\begin{array}{c}\text { MDA } \\
(\mathrm{nmol} / \mathrm{ml})\end{array}$ & $\begin{array}{c}\text { SOD } \\
(\mathrm{U} / \mathrm{ml})\end{array}$ \\
\hline Sham & $7.17 \pm 2.61$ & $186.10 \pm 16.23$ \\
MI/R & $17.01 \pm 3.58^{\mathrm{a}}$ & $69.66 \pm 16.32^{\mathrm{a}}$ \\
MI/R + DSS & $11.26 \pm 2.03^{\mathrm{b}}$ & $110.60 \pm 14.75^{\mathrm{b}}$ \\
MI/R + HSYA & $9.05 \pm 2.48^{\mathrm{b}}$ & $121.80 \pm 21.44^{\mathrm{b}}$ \\
MI/R + DH & $7.73 \pm 1.62^{\mathrm{c}}$ & $152.5 \pm 12.73^{\mathrm{c}}$ \\
\hline
\end{tabular}

The MDA and SOD activity levels were examined in the serum of rats in the sham-operated, MI/R, MI/R + DSS (60 mg/kg), MI/R + HSYA $(70 \mathrm{mg} / \mathrm{kg})$ and MI $/ \mathrm{R}+\mathrm{DSS}+\mathrm{HSYA}(35+35 \mathrm{mg} / \mathrm{kg})$ groups. Values are expressed as the means $\pm \mathrm{SD}$ ( $\mathrm{n}=7-9$ rats in each group). ${ }^{\mathrm{a}} \mathrm{P}<0.01$ vs. sham-operated (sham) group; ${ }^{\mathrm{P}} \mathrm{P}<0.05$ and ${ }^{\mathrm{c}} \mathrm{P}<0.01$ vs. MI/R group. MI/R, myocardial ischemiareperfusion; HSYA, hydroxysafflor yellow A; DSS, danshensu.

DSS and HSYA synergistically protect H9c2 cardiomyocytes against injury induced by $H / R$. The results of MTT assay demonstrated that $\mathrm{H} / \mathrm{R}$ significantly reduced $(\mathrm{P}<0.05$ vs. Con) cell viability to $35.26 \pm 6.10 \%$, while DSS and HSYA used alone or in combination protected the H9c2 cardiomyocytes against $\mathrm{H} / \mathrm{R}$ injury, and the $\mathrm{DH}$ group exhibited a significant increase in viability at dose 3 to $68.38 \pm 3.35 \%$ ( $\mathrm{P}<0.05$ vs. $\mathrm{H} / \mathrm{R}+\mathrm{DSS}$ or H/R + HSYA) (Fig. 4A). The dose-effect curves of DSS and HSYA used alone or in combination were analyzed using the median-effect method to determine whether they acted
Table IV. Number of rats that failed the coronary ligation procedure in each group shown in Fig. 3.

Group Sham MI/R MI/R + DSS MI/R + HSYA MI/R + DH

\begin{tabular}{llllll}
\hline No. & 2 & 2 & 3 & 3 & 1 \\
\hline
\end{tabular}

MI/R, myocardial ischemia-reperfusion; HSYA, hydroxysafflor yellow A; DSS, danshensu; DH, HYSA + DSS.

synergistically to protect the H9c2 cardiomyocytes against H/R injury (Fig. 4B-1). We found that treatment with DSS and HSYA or in combination at concentrations of $1-80 \mu \mathrm{M}$ yielded $\mathrm{CI}_{50}$ values $<1$ (Fig. 4B-2), indicating synergistic effects between the agents. Since the agents exerted cardioprotective effects in a dose-dependent manner, the highest doses were selected for use in subsequent studies. As an acknowledged marker of cell damage, the LDH levels in the cell supernatant significantly increased ( $\mathrm{P}<0.01$ vs. Con) to $91.14 \pm 9.71 \mathrm{U} / 1$ in the $\mathrm{H} / \mathrm{R}$ group. The DSS or HSYA groups exhibited levels of $53.50 \pm 12.54 \mathrm{U} / 1$ and $65.11 \pm 5.51 \mathrm{U} / 1$, respectively $(\mathrm{P}<0.05 \mathrm{vs}$. $\mathrm{H} / \mathrm{R})$. These effects were markedly enhanced in the DH group compared with the $\mathrm{H} / \mathrm{R}$ group $(\mathrm{P}<0.01$; Fig. $4 \mathrm{C})$.

DSS and HSYA increase HO- 1 expression in H9c2 cardiomyocytes. HO-1 expression was increased in the H/R + DSS group $(80 \mu \mathrm{M})$ and the $\mathrm{H} / \mathrm{R}+\mathrm{HSYA}$ group $(80 \mu \mathrm{M})$. HO-1 expression was further increased in the $\mathrm{H} / \mathrm{R}+\mathrm{DH}$ group $(40+40 \mu \mathrm{M})$ to a level higher than that in the single treat- 

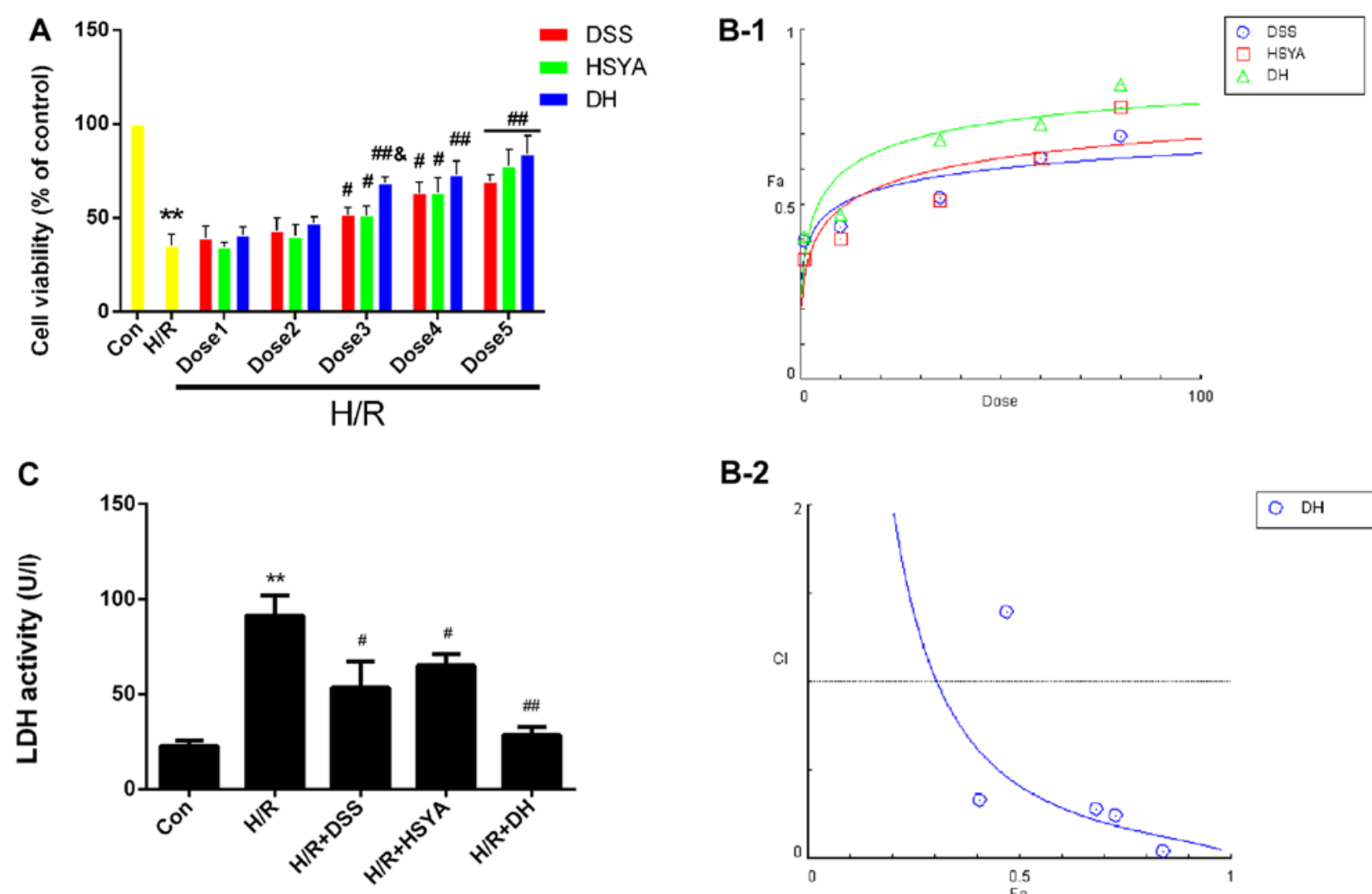

\section{B-2}

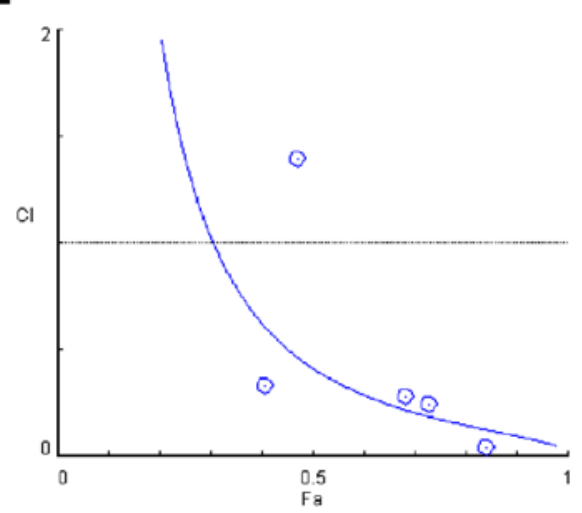

Figure 4. Danshensu (DSS), hydroxysafflor yellow A (HSYA) administered individually or in combination protect the H9c2 cardiomyocytes from hypoxia/reoxygentaion (H/R) injury, and in combitation, exert a synergistic effect. (A) Cell viability was detected by an MTT reduction assay. H9c2 cardiomyocytes in the control (Con) group were considered 100\% viable. Dose 1 indicates DSS $(1 \mu \mathrm{M})$, HSYA $(1 \mu \mathrm{M})$, DH $(1 \mu \mathrm{M})$; Dose 2 indicates DSS (10 $\mu \mathrm{M})$, HSYA (10 $\mu \mathrm{M})$, DH $(10 \mu \mathrm{M}$; Dose 3 indicates DSS $(35 \mu \mathrm{M})$, HSYA $(35 \mu \mathrm{M})$, DH $(35 \mu \mathrm{M})$; Dose 4 indicates DSS $(60 \mu \mathrm{M})$, HSYA $(60 \mu \mathrm{M})$, DH $(60 \mu \mathrm{M})$; Dose 5 indicates DSS $(80 \mu \mathrm{M})$, HSYA $(80 \mu \mathrm{M})$, DH $(80 \mu \mathrm{M})$. (B-1) The dose-effect curves of the single or combined drug treatment. (B-2) The fraction versus combination index (Fa-CI) curve of DSS and HSYA in combination reveals that they exert a synergistic effect $\left(\mathrm{CI}_{50}<1\right)$ as reflected by the median-effect method. The dashed line at the combination index $(\mathrm{CI}=1)$ represents the additive effect. (C) The effect of DSS and HSYA on LDH activity in the Con, H/R, H/R + DSS ( $80 \mu \mathrm{M})$, $\mathrm{H} / \mathrm{R}+\mathrm{HSYA}(80 \mu \mathrm{M})$ and $\mathrm{H} / \mathrm{R}+\mathrm{DH}(40+40 \mu \mathrm{M})$ groups. Values are expressed as the means $\pm \mathrm{SD}(\mathrm{n}=6) .{ }^{* *} \mathrm{P}<0.01$ vs. Con group; ${ }^{*} \mathrm{P}<0.05$ and ${ }^{* \#} \mathrm{P}<0.01 \mathrm{vs}$. $\mathrm{H} / \mathrm{R}$ group; ${ }^{\circledR} \mathrm{P}<0.05$ vs. $\mathrm{H} / \mathrm{R}+\mathrm{DSS}$ or $\mathrm{H} / \mathrm{R}+\mathrm{HSYA}$ group.

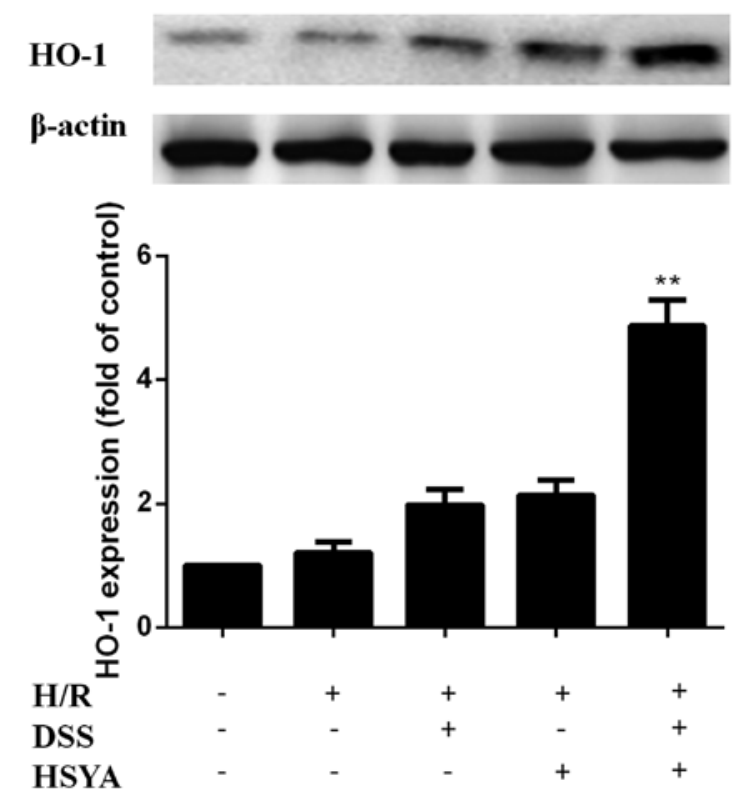

Figure 5. Heme oxygenase-1 (HO-1) expression in the control (Con), hypoxia/reoxygenation $(\mathrm{H} / \mathrm{R}), \mathrm{H} / \mathrm{R}+$ Danshensu $(\mathrm{DSS} ; 80 \mu \mathrm{M}), \mathrm{H} / \mathrm{R}+$ hydroxysafflor yellow A (HSYA; $80 \mu \mathrm{M})$ and H/R + DSS + HSYA $(40+40 \mu \mathrm{M})$ groups. Data obtained from half-quantitative densitometry are presented as the means \pm SD of 3 independent experiments. ${ }^{* *} \mathrm{P}<0.01$ vs. H/R + DSS or $\mathrm{H} / \mathrm{R}+\mathrm{HSYA}$ group. Note that cropped gel images are used in this figure and the gels were run under the same experimental conditions. ment groups $(\mathrm{P}<0.01)$. There were no significant differences between the $\mathrm{H} / \mathrm{R}$ group and the groups treated with each agent alone (Fig. 5).

The antioxidant effects of DSS and HSYA in H9c2 cardiomyocytes are negated by ZnPP-IX. The analysis of the MDA content and SOD activity revealed that H/R deteriorated the parameters of oxidative stress significantly compared with the control group $(\mathrm{P}<0.01)$. The MDA content markedly decreased to $2.47 \pm 0.44 \mathrm{nmol} / \mathrm{mlintheDSS}$ groupand to $2.05 \pm 0.53 \mathrm{nmol} / \mathrm{ml}$ in the HSYA group $(\mathrm{P}<0.05$ vs. $\mathrm{H} / \mathrm{R})$, and the activity of SOD increased to $11.06 \pm 1.64$ and $13.53 \pm 2.30 \mathrm{nmol} / \mathrm{ml}$ in the DSS and the HSYA group, respectively. These protective effects were more significantly enhanced in the DH group compared with the H/R group (MDA, $\mathrm{P}<0.01$; SOD, $\mathrm{P}<0.05$ ). HSYA also exerted a more potent antioxidant effect than DSS. The antioxidant effects were markedly abrogated by ZnPP-IX ( $\mathrm{P}<0.05$ vs. H/R + DH) (Table V).

ZnPP-IX inhibits the anti-apoptotic effect of DSS and HSYA in H9c2 cardiomyocytes. TUNEL staining of apoptotic cells demonstrated that H/R led to a significant augmentation in cell apoptosis to $56.41 \pm 14.23 \%$ 
A
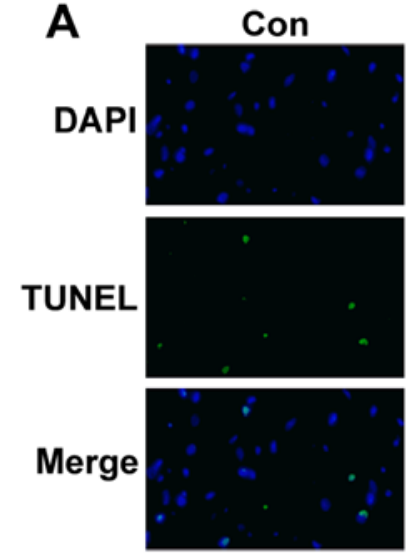

$H / R$
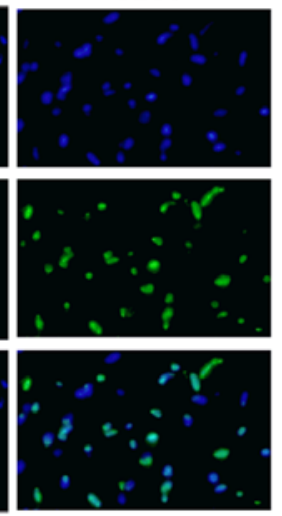

H/R+DSS
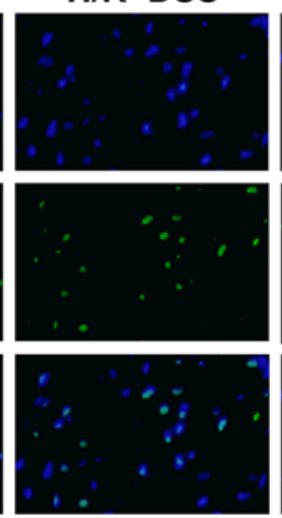

H/R+HSYA
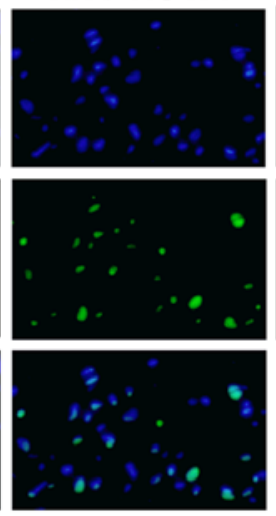

$\mathrm{H} / \mathrm{R}+\mathrm{DH}$
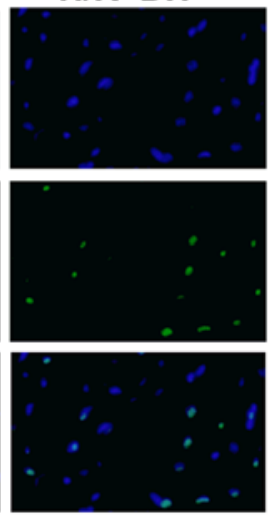

$\mathrm{H} / \mathrm{R}+\mathrm{DH}+\mathrm{Z}$
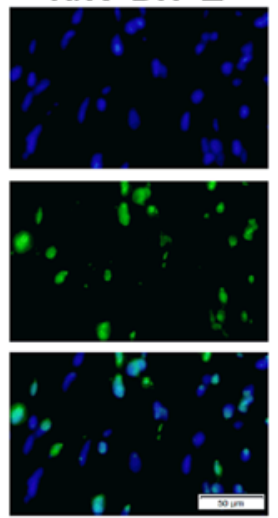

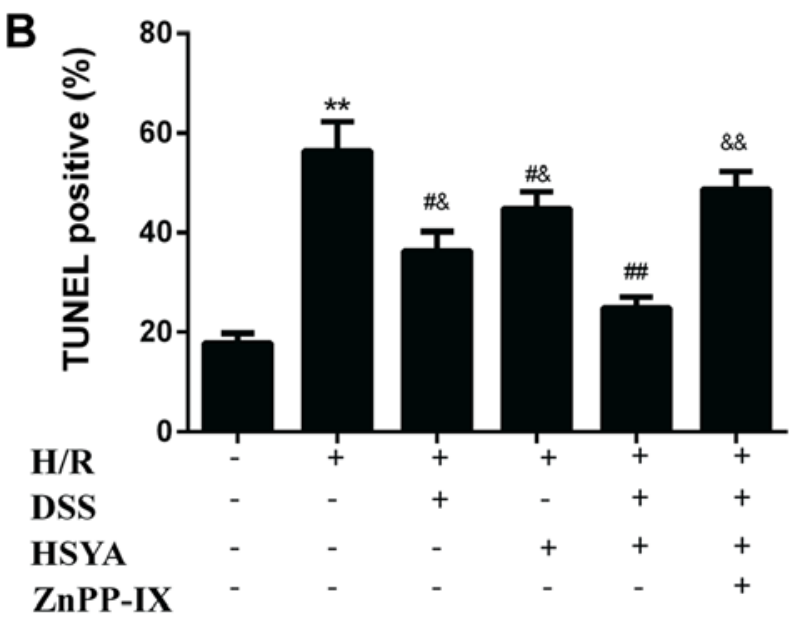

Figure 6. Representative images of the TUNEL staining in the control (Con), hypoxia/reoxygenation (H/R), H/R + Danshensu (DSS; $80 \mu \mathrm{M}), \mathrm{H} / \mathrm{R}+\mathrm{hydroxysafflor}$ yellow A (HSYA; $80 \mu \mathrm{M})$ and H/R + DSS + HSYA $(40+40 \mu \mathrm{M})$ groups. (A) (Top panel) DAPI staining (blue) indicates total nuclei, (middle panel) apoptotic nuclei detected by TUNEL staining (green), and (bottom panel) overlay of both types of staining. (B) The number of TUNEL-positive myocytes was expressed as a percentage of total nuclei detected by DAPI staining (fluorescence microscopy, magnification, $\mathrm{x} 40$, scale bar, $50 \mu \mathrm{m}$ ) Data are presented as the means \pm SD of 3 independent experiments. ${ }^{* *} \mathrm{P}<0.01$ vs. Con group; ${ }^{*} \mathrm{P}<0.05$ and ${ }^{\# \#} \mathrm{P}<0.01$ vs. $\mathrm{H} / \mathrm{R}$ group; ${ }^{\&} \mathrm{P}<0.05$ and ${ }^{\&} \mathrm{P}<0.01$ vs. $\mathrm{H} / \mathrm{R}+\mathrm{DH}$ group.

Table V. MDA content and SOD activity in culture supernatants of cardiomyocytes.

\begin{tabular}{lcc}
\hline Treatment & $\begin{array}{c}\text { MDA } \\
(\mathrm{nmol} / \mathrm{ml})\end{array}$ & $\begin{array}{c}\text { SOD } \\
(\mathrm{U} / \mathrm{ml})\end{array}$ \\
\hline Con & $1.72 \pm 0.70$ & $17.98 \pm 2.84$ \\
$\mathrm{H} / \mathrm{R}$ & $4.01 \pm 0.85^{\mathrm{a}}$ & $7.40 \pm 2.90^{\mathrm{a}}$ \\
$\mathrm{H} / \mathrm{R}+\mathrm{DSS}$ & $2.47 \pm 0.44$ & $11.06 \pm 1.64$ \\
$\mathrm{H} / \mathrm{R}+\mathrm{HSYA}$ & $2.05 \pm 0.53^{\mathrm{b}}$ & $13.53 \pm 2.30$ \\
$\mathrm{H} / \mathrm{R}+\mathrm{DH}$ & $1.74 \pm 0.63^{\mathrm{c}}$ & $15.58 \pm 3.04^{\mathrm{b}}$ \\
$\mathrm{H} / \mathrm{R}+\mathrm{DH}+\mathrm{Z}$ & $3.62 \pm 0.42^{\mathrm{d}}$ & $7.11 \pm 2.64^{\mathrm{d}}$ \\
\hline
\end{tabular}

The MDA content and SOD activity were calculated in the cardiomyocytes in the Con, H/R, H/R + DSS $(80 \mu \mathrm{M}), \mathrm{H} / \mathrm{R}+\mathrm{HSYA}(80 \mu \mathrm{M})$ and $\mathrm{H} / \mathrm{R}+\mathrm{DSS}+\mathrm{HSYA}(40+40 \mu \mathrm{M})$ groups. Values are expressed as the means $\pm \mathrm{SD}(\mathrm{n}=6) .{ }^{\mathrm{P}} \mathrm{P}<0.01$ vs. Con group; ${ }^{\mathrm{b}} \mathrm{P}<0.05$ and ${ }^{\mathrm{C}} \mathrm{P}<0.01 \mathrm{vs}$. $\mathrm{H} / \mathrm{R}$ group; ${ }^{\mathrm{d}} \mathrm{P}<0.05$ vs. $\mathrm{H} / \mathrm{R}+\mathrm{DH}$ group. $\mathrm{H} / \mathrm{R}$, hypoxia/reoxygenation, HSYA, hydroxysafflor yellow A; DSS, danshensu; MDA, malondialdehyde; SOD, superoxide dismutase; Z, zinc protoporphyrin IX; Con, control.

$(\mathrm{P}<0.01$ vs. Con). Apoptosis was alleviated in the group treated with DSS or HSYA to $36.38 \pm 9.46$ and $44.88 \pm 8.10 \%$, respectively $(\mathrm{P}<0.05$ vs. $\mathrm{H} / \mathrm{R})$. Apoptosis was decreased in the DH group to $24.95 \pm 5.02 \%(\mathrm{P}<0.05$ vs. $\mathrm{H} / \mathrm{R}+\mathrm{DSS}$ or H/R + HSYA). ZnPP-IX abrogated the anti-apoptotic effect of DSS and HSYA (P<0.01 vs. H/R + DH) (Fig. 6). Following $20 \mathrm{~h}$ of reoxygenation, the $\mathrm{Bcl}-2 / \mathrm{Bax}$ ratio decreased to $0.58 \pm 0.34$ in the H/R-exposed cardiomyocytes. DSS or HSYA reversed the ratio to $2.86 \pm 0.95$ and $1.93 \pm 0.49$, respectively $(\mathrm{P}<0.01$ and $\mathrm{P}<0.05$ vs. H/R). In addition, treatment with $\mathrm{DH}$ increased the $\mathrm{Bcl}-2 / \mathrm{Bax}$ ratio to $4.93 \pm 0.60(\mathrm{P}<0.01$ vs. $\mathrm{H} / \mathrm{R}+\mathrm{DSS}$ or $\mathrm{H} / \mathrm{R}+\mathrm{HSYA})$. The $\mathrm{Bcl}-2 / \mathrm{Bax}$ ratio was downregulated in the group treated with ZnPP-IX to $1.41 \pm 0.32(\mathrm{P}<0.01$ vs. H/R + DH) (Fig. 7A). H/R evoked a marked increase in the cleaved caspase-3 levels to $10.74 \pm 1.39$ ( $\mathrm{P}<0.01$ vs. Con), while DSS or HSYA decreased cleaved caspase-3 expression to $3.74 \pm 1.34$ and $5.46 \pm 1.90$, respectively ( $\mathrm{P}<0.01 \mathrm{vs}$. H/R). Treatment with DH decreased the cleaved caspase-3 level to $1.80 \pm 0.85$ ( $\mathrm{P}<0.01$ vs. H/R + DSS or H/R + HSYA), and in the group co-incubated with ZnPP-IX, this effect was abolished $(8.87 \pm 2.82 ; \mathrm{P}<0.01$ vs. H/R + DH) (Fig. 7B). Furthermore, we found that DSS exerted a more potent anti-apoptotic effect than HSYA.

DSS and HSYA modulate the acvitation of the Akt/Nrf2/HO-1 signaling pathway in H9c2 cardiomyocytes. Following $20 \mathrm{~h}$ 
A
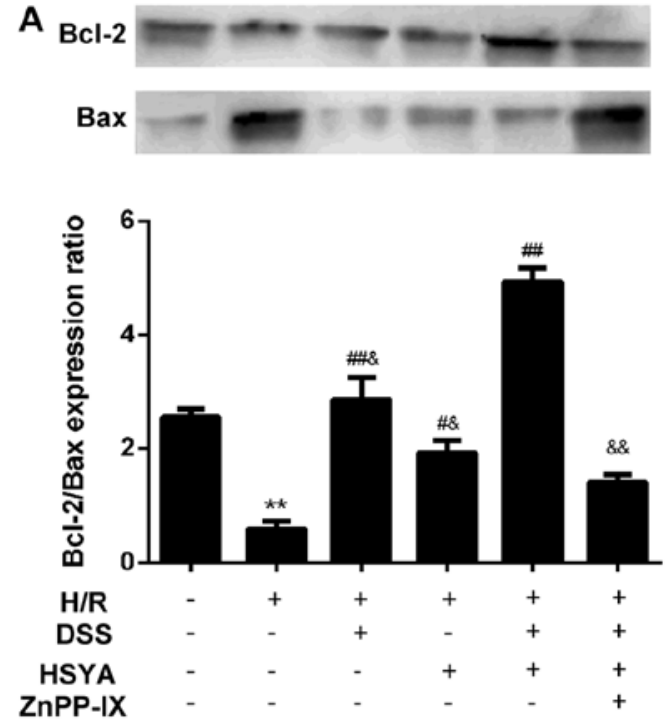
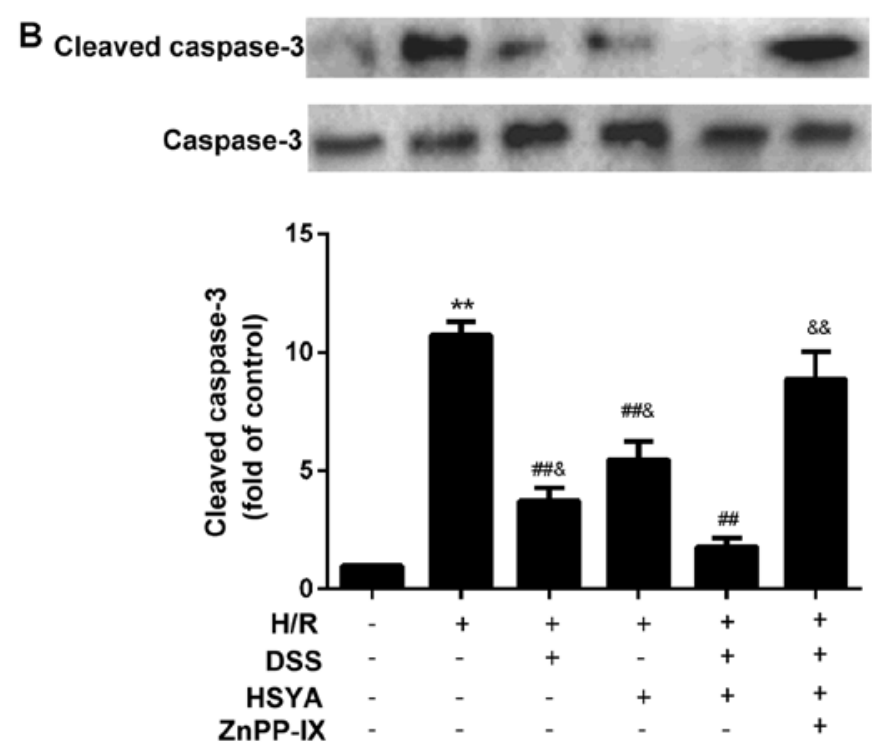

Figure 7. Bcl-2, Bax and cleaved caspase-3 levels in the control (Con), hypoxia/reoxygenation (H/R), H/R + Danshensu (DSS; $80 \mu \mathrm{M}), \mathrm{H} / \mathrm{R}+\mathrm{hydroxysafflor}$ yellow A (HSYA; $80 \mu \mathrm{M}), \mathrm{H} / \mathrm{R}+\mathrm{DSS}+\mathrm{HSYA}(40+40 \mu \mathrm{M})$ and H/R + DH + zinc protoporphyrin IX (ZnPP-IX; $10 \mu \mathrm{M})$ groups. (A) Bcl-2/Bax was calculated and data are presented as the means \pm SD of 3 independent experiments; (B) cleaved caspase-3 levels obtained from half-quantitative densitometry are presented as the means $\pm \mathrm{SD}$ of 3 independent experiments. ${ }^{* *} \mathrm{P}<0.01$ vs. Con group; ${ }^{\#} \mathrm{P}<0.05$ and ${ }^{\# \#} \mathrm{P}<0.01$ vs. $\mathrm{H} / \mathrm{R}$ group; ${ }^{\&} \mathrm{P}<0.05$ and ${ }^{\&} \& \mathrm{P}<0.01$ vs. $\mathrm{H} / \mathrm{R}+\mathrm{DH}$ group. Note that cropped gel images are used in this figure and the gels were run under the same experimental conditions.

of reoxygenation, treatment with DSS or HSYA increased the expression of p-Akt, HO-1 and nuclear Nrf2, and combined treatment exerted even more significant effects than treatment with each agent alone $(\mathrm{P}<0.01)$. LY294002 markedly abolished the effects of DSS and HSYA on p-Akt expression $(\mathrm{P}<0.01 \mathrm{vs} . \mathrm{H} / \mathrm{R}+\mathrm{DH})$, and partly blocked the expression of nuclear Nrf2 and HO-1 ( $\mathrm{P}<0.05$ and $\mathrm{P}<0.01$ vs. $\mathrm{H} / \mathrm{R}+\mathrm{DH})$. ZnPP-IX had no significant effect on p-Akt and nuclear Nrf2 expression compared with the $\mathrm{H} / \mathrm{R}+\mathrm{DH}$ group; however, it markedly negated HO-1 expression ( $\mathrm{P}<0.01$ vs. $\mathrm{H} / \mathrm{R}+\mathrm{DH})$ (Fig. 8).

\section{Discussion}

A number of studies have illustrated the protective effects of DSS or HSYA in cardiovascular diseases $(15,16,18)$; however, limited attention has been paied to their use as a combination therapy and the mechanisms responsible for their combined effects have yet to be elucidated. In the present study, we investigated the synergistic protective effects of DSS and HSYA on myocardial injury through in vivo experiments using rats and in vitro experiments using $\mathrm{H} 9 \mathrm{c} 2$ cardiomyocytes. To the best of our knowledge, this is the first study to demonstrate the role of DSS and HSYA in combination to protect against cellular injury through antioxidant and anti-apoptotic effects, which are associated with the activation of the Akt/Nrf2/HO-1 signaling pathway.

Infarct size is regarded as the gold standard in assessing the severity of MI/R injury (25). Thus, the first investigations of the combination effects examined infarct size. Following the administration of a series of doses of DSS and/or HSYA, the infarct size was reduced in a dose-dependent manner. The $\mathrm{CI}_{50}$ value of the infarct size $(<1)$ determined by the medianeffect method verified the combination effects to be synergistic between the agents. Additionally, the sensitive cardiac injury markers (26-28) CK-MB and cTnI, were measured to determine whether DSS and HSYA are capable of alleviating the degree of myocardial injury caused by MI/R. The decreased release of CK-MB and cTnI in the treatment groups demonstrated the protective effects of DSS and HSYA. Furthermore, combined treatment exerted a more potent protective effect compared to treatment with either agent alone. To further confirm the combination effects, an MTT assay was performed. The $\mathrm{CI}_{50}$ value of cell viability was revealed to be $<1$, indicating a synergistic effect between DSS and HSYA. LDH is one of the specific enzymes (29) present in the myocardial cytoplasm, and its values may indirectly reflect the degree of damage of the myocardium exposed to H/R. In the present study, the LDH levels in the cell supernatant were significantly decreased following treatment with $\mathrm{DH}$. These results indicated that DSS and HSYA used in combination exerted a synergistic cardioprotective effect in vitro and in vivo.

Having determined that DSS and HSYA exerted a synergistic cardioprotective effect, we then proceeded to elucidate the possible mechanisms responsible for these effects. The increased production of oxygen-free radicals in conjunction with the decreased activity of antioxidant defenses are considered to be an significant factor for myocardial reperfusion injury (30). SOD is the first line of cellular defense against oxidative injury, which decomposes $\mathrm{O}_{2}$ and $\mathrm{H}_{2} \mathrm{O}_{2}$ before they interact to form the more reactive hydroxyl radical $(31,32)$. MDA is a product of lipid peroxidation that may cause the crosslinking polymerization of proteins, nucleic acids and some macromolecules, and thus, it has been found that the amount of MDA often reflects the degree of lipid peroxidation (33). 8-OHdG appears to be a sensitive and integral marker of oxidative damage to DNA due to any imbalance between $\mathrm{OH}^{\bullet}$ generation, antioxidant defenses and the repair of damaged DNA sequences (34). Using these three important markers of oxidative stress, we confirmed that DSS and HSYA significantly alleviated the parameters of oxidative stress and HSYA exerted a profound protective effect 
A
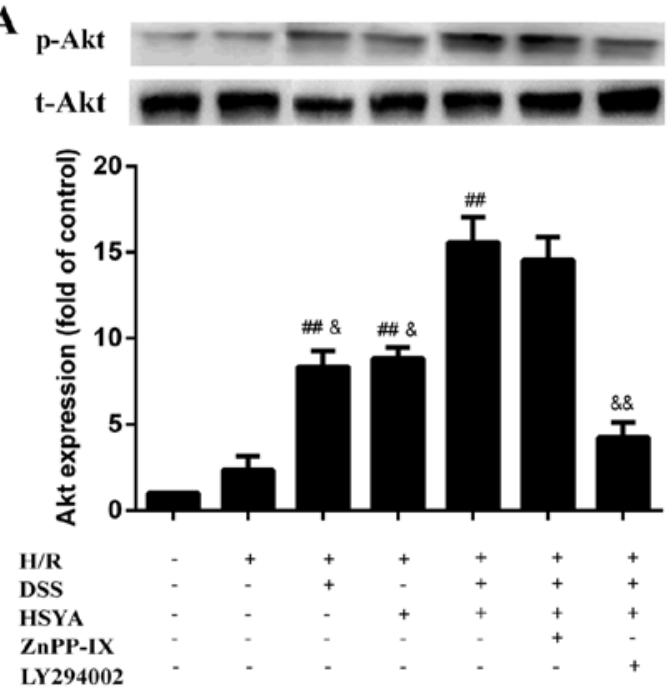
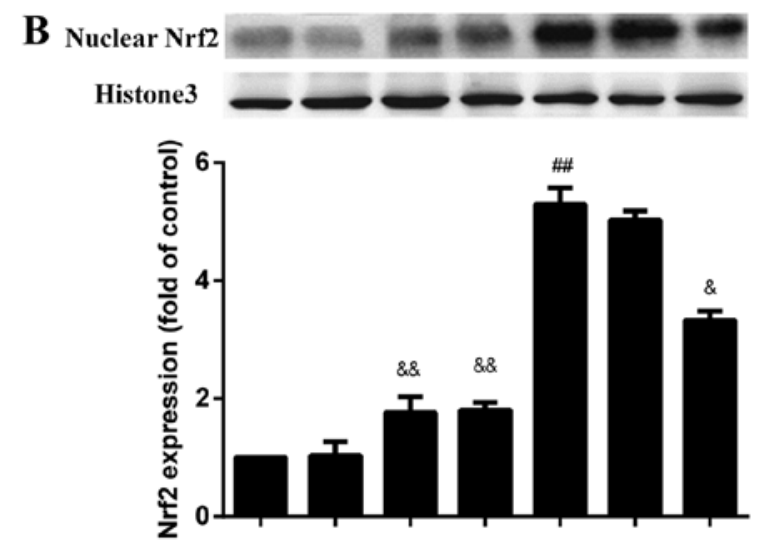

$\begin{array}{llllllll}\text { H/R } & - & + & + & + & + & + & + \\ \text { DSS } & - & - & + & - & + & + & + \\ \text { HSYA } & - & - & - & + & + & + & + \\ \text { ZnPP-IX } & - & - & - & - & - & + & - \\ \text { LY294002 } & - & - & - & - & - & - & +\end{array}$
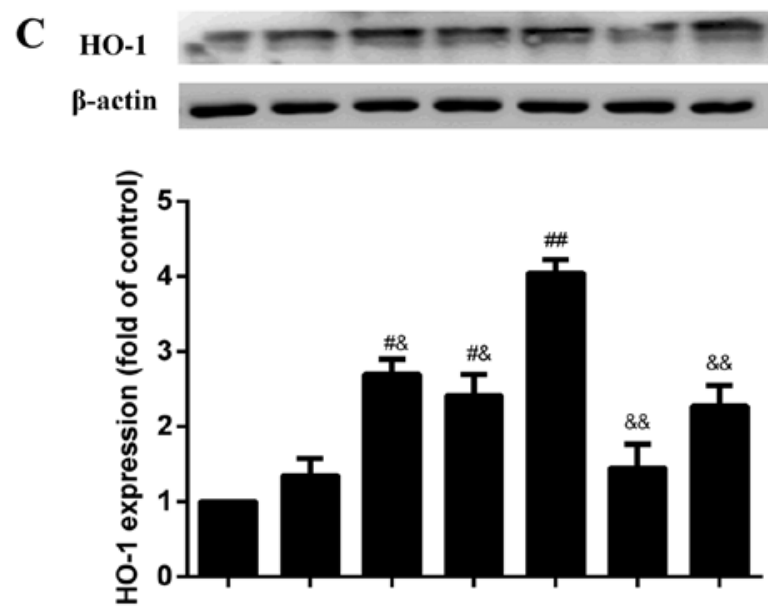

$\begin{array}{llllllll}\text { H/R } & - & + & + & + & + & + & + \\ \text { DSS } & - & - & + & - & + & + & + \\ \text { HSYA } & - & - & - & + & + & + & + \\ \text { ZnPP-IX } & - & - & - & - & - & + & - \\ \text { LY294002 } & - & - & - & - & - & - & +\end{array}$

Figure 8. (A) Protein kinase B (Akt), (B) nuclear factor erythroid 2-related factor 2 (Nrf2) and (C) heme oxygenase-1 (HO-1) levels in the control (Con), hypoxia/reoxygenation (H/R), H/R + Danshensu (DSS; $80 \mu \mathrm{M}), \mathrm{H} / \mathrm{R}+$ hydroxysafflor yellow A (HSYA; $80 \mu \mathrm{M}), \mathrm{H} / \mathrm{R}+\mathrm{DSS}+\mathrm{HSYA}(40+40 \mu \mathrm{M})$, $\mathrm{H} / \mathrm{R}+\mathrm{DH}+$ zinc protoporphyrin IX (ZnPP-IX; $10 \mu \mathrm{M})$ and $\mathrm{H} / \mathrm{R}+\mathrm{DH}+\mathrm{LY} 294002(50 \mu \mathrm{M})$ groups. Data obtained from half-quantitative densitometry are presented as the means $\pm \mathrm{SD}$ of 3 independent experiments. ${ }^{\#} \mathrm{P}<0.05$ and ${ }^{\# \#} \mathrm{P}<0.01 \mathrm{vs}$. $\mathrm{H} / \mathrm{R}$ group; ${ }^{\circledR} \mathrm{P}<0.05$ and ${ }^{\text {\&\&}} \mathrm{P}<0.01 \mathrm{vs}$. H/R $+\mathrm{DH}$ group. Note that cropped gel images are used in this figure and the gels were run under the same experimental conditions. t-AKT, total AKT; p-AKT, phospho-AKT.

in vitro and in vivo. This finding indicated that DSS and HSYA played a pivotal role in ameliorating the oxidative stress injury caused by $\mathrm{MI} / \mathrm{R}$ or $\mathrm{H} / \mathrm{R}$.

$\mathrm{HO}-1$ is an inducible enzyme with potent antioxidant activities due to its ability to degrade heme into biliverdin or bilirubin, carbon monoxide and free iron $(35,36)$. Furthermore, it has been demonstrated that plant-derived chemical substances may act as inducers of the HO-1, and these substances have been found to exert cardioprotective effects against oxidative injury (37). Thus, we hypothesized that DSS and HSYA may protect myocardial tissue and H9c2 cardiomyocytes from oxidative stress-induced injury in association with the upregulation of HO-1. Western blot analysis revealed that HO-1 expression was profoundly enhanced in the combined treatment group compared with the groups treated with either agent alone. This result not only verified the above-mentioned hypothesis, but also indicated that combination therapy may be more effective in increasing HO-1 expression than monotherapy. It has been noted that ZnPP-IX, which is a competitive inhibitor of the HO-1 enzyme, conjugates to HO-1 and reduces the conjugation between HO-1 and heme $(38,39)$. Following treatment with ZnPP-IX $(10 \mu \mathrm{M})$, the decreased MDA content and the increased activity of SOD were both revoked. These results illustrated that DSS and HSYA upregulated HO-1 expression and that the antioxidant effects were partly dependent on HO-1 expression.

Apoptosis plays an important role in the loss of cardiomyocytes in a variety of pathologies, including $H / R$ injury $(18,40)$. It has been demonstrated that the mitochondrial apoptotic pathway may be activated when a number of pro-apoptotic factors are released (41). Bax is a pro-apoptotic protein, whereas $\mathrm{Bcl}-2$ is an anti-apoptotic protein. It has been noted that the ratio of Bcl-2 to Bax acts as an essential element in determining the threshold of apoptosis $(18,42)$. Caspases transduce and execute 
apoptotic signaling. Caspase-3 has been found to be activated by the apoptotic pathway and then autocatalyzed, and processed into activated fragments such as cleaved caspase-3, which are considered as an index of apoptosis (43). In the present study, DSS and HSYA increased the Bcl-2/Bax ratio in the mitochondria, downregulated cleaved caspase-3 expression and reduced TUNEL staining compared with the H/R group. DH therapy enhanced the effect to a greater extent compared with DSS or HSYA alone. The anti-apoptotic effects were markedly negated by ZnPP-IX. Additionally, DSS exerted a more potent anti-apoptotic effect than HSYA. These results confirmed that the overexpression of HO-1 induced by the agents and the combined regimen led to an enhanced anti-apoptotic effect.

It has been proven that a specific molecular pathway is important for compound-induced HO-1 expression. The PI3K/Akt pathway has been demonstrated to be associated with increased HO-1 expression (44). In this study, Akt phosphorylation was significantly enhanced in the H9c2 cardiomyocytes treated with DSS and HSYA compared with that observed in the H/R group. The Akt inhibitor, LY294002, significantly blocked this effect, while ZnPP-IX had no obvious impact on Akt phosphorylation induced by the two agents. Nrf2 is a key regulator of HO-1 expression in cells (45). It has been demonstrated that the nuclear factor Nrf2 binds to Kelch-like ECH-associated protein-1 (Keap1) to form the Keap1-Nrf2 complex and limit Nrf2-mediated gene expression in the cytoplasm under normal physiological circumstances. Nrf2 is released from Keapl under conditions of oxidative stress or other potential damage and then translocates to the nucleus, where it binds to antioxidant response element (ARE) sequences, leading to the transcriptional activation of anti-apoptotic genes, including HO-1 (18,46-49). In the present study, the two agents enhanced the expression of nuclear Nrf2. LY294002 partly blocked the increase in Nrf2 translocation to the nucleus and the upregulation of HO-1 expression, while ZnPP-IX had no notable effect on nuclear Nrf2 expression induced by DSS and HSYA. These results indicated that the DSS- and HSYA-mediated upregulation of Akt phosphorylation and nuclear Nrf2 did not rely on the upregulation of HO-1 expression. On the contrary, the upregulation of HO-1 expression was dependent on Akt phosphorylation. Akt phosphorylation, as well as nuclear Nrf2 and HO-1 expression were enhanced in the DH-treated group compared with that observed in the groups treated with either agent alone. As we confirmed above, treatment with DH exerted a synergistic effect. However, it would be interesting to determine the possible mechanisms underlying these synergistic effects. It has been pointed out that each single constituent of a combination affects several targets, such as enzymes, substrates, receptors and transport proteins $(8,50)$. Most notably, DSS mainly exerted anti-apoptotic effects, while HSYA exerted significant antioxidant effects. They acted on apoptosis-related proteins and oxidative-related enzymes, respectively; thus, in combination they exerted complementary and synergistic effects. Our results revealed that the combination of DSS and HSYA conferred a synergistic effect on the activation of the Akt/Nrf2/HO-1 pathway, which is a potential mechanism for enhancing the anti-apoptotic and antioxidant effects.

In the present study, we only investigated the antioxidant and anti-apoptotic effects involving the Akt/Nrf2/HO-1 pathway. It would be of interest to determine whether other aspects contribute to these synergistic effects. Further studies are warranted to clarify the synergyistic effects of DSS and HSYA on the regulation of other pathways.

In conclusion, we found that DSS and HSYA acted synergistically to significantly attenuate myocardial injury in vitro and in vivo by exerting antioxidant and anti-apoptotic effects through the Akt/Nrf2/HO-1 signaling pathway. The results from the present study provide insight into the effects and the mechanisms responsible for these synergistic effects. This may lead to the development of effective combined therapeutic TCM regimens so as to combat myocardial complications in clinical practice.

\section{Acknowledgements}

This study was financially supported by the National Natural Science Foundation of China (nos. 81173514, 81403135, 81403182 and 81403134 ). The authors would like to thank their colleagues at the New Drug Research and Development Center of Xijing Hospital and the staff of China National Corp. of Traditional and Herbal Medicine for their technical assistance.

\section{References}

1. Bainey KR and Armstrong PW: Clinical perspectives on reperfusion injury in acute myocardial infarction. Am Heart $\mathrm{J}$ 167: 637-645, 2014

2. Maxwell SR and Lip GY: Reperfusion injury: a review of the pathophysiology, clinical manifestations and therapeutic options. Int J Cardiol 58: 95-117, 1997.

3. Yellon DM and Hausenloy DJ: Myocardial reperfusion injury. N Engl J Med 357: 1121-1135, 2007.

4. Buja LM: Myocardial ischemia and reperfusion injury. Cardiovasc Pathol 14: 170-175, 2005.

5. Olivetti G, Quaini F, Sala R, Lagrasta C, Corradi D, Bonacina E, Gambert SR, Cigola E and Anversa P: Acute myocardial infarction in humans is associated with activation of programmed myocyte cell death in the surviving portion of the heart. $\mathrm{J}$ Mol Cell Cardiol 28: 2005-2016, 1996.

6. Zhao ZQ, Nakamura M, Wang NP, Wilcox JN, Shearer S, Ronson RS, Guyton RA and Vinten-Johansen J: Reperfusion induces myocardial apoptotic cell death. Cardiovasc Res 45: 651-660, 2000 .

7. Hori $\mathrm{M}$ and Nishida $\mathrm{K}$ : Oxidative stress and left ventricular remodelling after myocardial infarction. Cardiovasc Res 81: 457-464, 2009.

8. Wagner H: Synergy research: approaching a new generation of phytopharmaceuticals. Fitoterapia 82: 34-37, 2011.

9. Combination Pharmacotherapy and Public Health Research Working Group: Combination pharmacotherapy for cardiovascular disease. Ann Intern Med 143: 593-599, 2005.

10. Wang L, Zhou GB, Liu P, Song JH, Liang Y, Yan XJ, Xu F, Wang BS, Mao JH, Shen ZX, et al: Dissection of mechanisms of Chinese medicinal formula Realgar-Indigo naturalis as an effective treatment for promyelocytic leukemia. Proc Natl Acad Sci USA 105: 4826-4831, 2008.

11. Li SJ, Tang YP, Shen J, Li JP, Guo JM and Duan JA: Research of Chinese medicine pairs (VIII) - Salviae Miltiorrhizae Radix et Rhizoma-Carthami flos. Zhongguo Zhong Yao Za Zhi 38: 4227-4231, 2013 (In Chinese).

12. Guan Y, Yin Y, Zhu YR, Guo C, Wei G, Duan JL, Wang YH, Zhou D, Quan W, Weng Y, et al: Dissection of mechanisms of a chinese medicinal formula: Danhong injection therapy for myocardial ischemia/reperfusion injury in vivo and in vitro. Evid Based Complement Alternat Med 2013: 972370, 2013.

13. Zhou D, Zhu YR, Guan Y, Quan W, Guo C, Weng Y, Cui J, Chen L, Ding Y, Xi M and Wen A: Development of chemical fingerprint and metabolic fingerprint of Danhong injection by HPLC-UV and HPLC-MS. Asian J Chem 25: 6285-6292, 2013. 
14. Duan JL, Wang JW, Guan Y, Yin Y, Wei G, Cui J, Zhou D, Zhu YR, Quan W, Xi MM and Wen AD: Safflor yellow A protects neonatal rat cardiomyocytes against anoxia/reoxygenation injury in vitro. Acta Pharmacol Sin 34: 487-495, 2013.

15. Yin Y, Guan Y, Duan J, Wei G, Zhu Y, Quan W, Guo C, Zhou D, Wang Y, Xi M and Wen A: Cardioprotective effect of Danshensu against myocardial ischemia/reperfusion injury and inhibits apoptosis of H9c2 cardiomyocytes via Akt and ERK1/2 phosphorylation. Eur J Pharmacol 699: 219-226, 2013.

16. Zhou MX, Fu JH, Zhang Q and Wang JQ: The effect of hydroxy safflower yellow A on inflammatory reaction in myocardium of the rats after acute myocardial infarction. Afr J Pharm Pharmacol 7: 643-649, 2013.

17. Cui G, Shan L, Hung M, Lei S, Choi I, Zhang Z, Yu P, Hoi P, Wang Y and Lee SM: A novel Danshensu derivative confers cardioprotection via PI3K/Akt and Nrf2 pathways. Int J Cardiol 168: 1349-1359, 2013.

18. Liu SX, Zhang Y, Wang YF, Li XC, Xiang MX, Bian C and Chen P: Upregulation of heme oxygenase-1 expression by hydroxysafflor yellow A conferring protection from anoxia/reoxygenation-induced apoptosis in $\mathrm{H} 9 \mathrm{c} 2$ cardiomyocytes. Int $\mathrm{J}$ Cardiol 160: 95-101, 2012.

19. Kin H, Zhao ZQ, Sun HY, Wang NP, Corvera JS, Halkos ME, Kerendi F, Guyton RA and Vinten-Johansen J: Postconditioning attenuates myocardial ischemia-reperfusion injury by inhibiting events in the early minutes of reperfusion. Cardiovasc Res 62: $74-85,2004$

20. Sachdeva J, Dai W, Gerczuk PZ and Kloner RA: Combined remote perconditioning and postconditioning failed to attenuate infarct size and contractile dysfunction in a rat model of coronary artery occlusion. J Cardiovasc Pharmacol Ther 19: 567-573, 2014

21. Mao X, Wang T, Liu Y, Irwin MG, Ou JS, Liao XL, Gao X, $\mathrm{Xu} \mathrm{Y}, \mathrm{Ng} \mathrm{KF}$, Vanhoutte PM and Xia Z: N-acetylcysteine and allopurinol confer synergy in attenuating myocardial ischemia injury via restoring $\mathrm{HIF}-1 \alpha / \mathrm{HO}-1$ signaling in diabetic rats. PLoS One 8: e68949, 2013.

22. Tsai CY, Wang CC, Lai TY, Tsu HN, Wang CH, Liang HY and Kuo WW: Antioxidant effects of diallyl trisulfide on high glucoseinduced apoptosis are mediated by the PI3K/Akt-dependent activation of Nrf2 in cardiomyocytes. Int J Cardiol 168: 1286-1297, 2013.

23. Chou TC: Drug combination studies and their synergy quantification using the Chou-Talalay method. Cancer Res 70: 440-446, 2010.

24. Won MH, Kang TC, Jeon GS, Lee JC, Kim DY, Choi EM, Lee KH, Choi CD, Chung MH and Cho SS: Immunohistochemical detection of oxidative DNA damage induced by ischemiareperfusion insults in gerbil hippocampus in vivo. Brain Res 836 70-78, 1999.

25. Panteghini M, Cuccia C, Bonetti G, Giubbini R, Pagani F and Bonini E: Single-point cardiac troponin $\mathrm{T}$ at coronary care unit discharge after myocardial infarction correlates with infarct size and ejection fraction. Clin Chem 48: 1432-1436, 2002.

26. Turer AT, Mahaffey KW, Gallup D, Weaver WD, Christenson RH, Every NR and Ohman EM: Enzyme estimates of infarct size correlate with functional and clinical outcomes in the setting of ST-segment elevation myocardial infarction. Curr Control Trials Cardiovasc Med 6: 12, 2005.

27. Christenson RH, Vollmer RT, Ohman EM, Peck S, Thompson TD, Duh SH, Ellis SG, Newby LK, Topol EJ and Califf RM; TAM Study Group: Relation of temporal creatine kinase-MB release and outcome after thrombolytic therapy for acute myocardial infarction. Am J Cardiol 85: 543-547, 2000.

28. Thygesen K, Alpert JS, White HD, Jaffe AS, Apple FS, Galvani M, Katus HA, Newby LK, Ravkilde J, Chaitman B, et al; Joint ESC/ACCF/AHA/WHF Task Force for the Redefinition of Myocardial Infarction: Universal definition of myocardial infarction. Eur Heart J 28: 2525-2538, 2007.

29. Quan W, Yin Y, Xi M, Zhou D, Zhu Y, Guan Y, Guo C, Wang Y, Duan J and Wen A: Antioxidant properties of magnesium lithospermate B contribute to the cardioprotection against myocardial ischemia/reperfusion injury in vivo and in vitro. $\mathbf{J}$ Tradit Chin Med 33: 85-91, 2013.

30. Das DK, Engelman RM, Rousou JA, Breyer RH, Otani H and Lemeshow S: Pathophysiology of superoxide radical as potential mediator of reperfusion injury in pig heart. Basic Res Cardiol 81: $155-166,1986$.
31. Wu J, Hecker JG and Chiamvimonvat N: Antioxidant enzyme gene transfer for ischemic diseases. Adv Drug Deliv Rev 61: 351-363, 2009.

32. Wattanapitayakul SK and Bauer JA: Oxidative pathways in cardiovascular disease: Roles, mechanisms, and therapeutic implications. Pharmacol Ther 89: 187-206, 2001.

33. Günfer T, Yaşar E and Bünyamin K: Changes in the levels of MDA and GSH in mice serum, liver and spleen after aluminum administration. East J Med 11: 7-12, 2006.

34. Cordis GA, Maulik G, Bagchi D, Riedel W and Das DK Detection of oxidative DNA damage to ischemic reperfused rat hearts by 8-hydroxydeoxyguanosine formation. J Mol Cell Cardiol 30: 1939-1944, 1998

35. Zeng B, Chen H, Zhu C, Ren X, Lin G and Cao F: Effects of combined mesenchymal stem cells and heme oxygenase-1 therapy on cardiac performance. Eur J Cardiothorac Surg 34: 850-856, 2008.

36. Tang YL, Tang Y, Zhang YC, Qian K, Shen L and Phillips MI Improved graft mesenchymal stem cell survival in ischemic heart with a hypoxia-regulated heme oxygenase-1 vector. J Am Coll Cardiol 46: 1339-1350, 2005.

37. Wu CC, Hsu MC, Hsieh CW, Lin JB, Lai PH and Wung BS: Upregulation of heme oxygenase-1 by epigallocatechin-3-gallate via the phosphatidylinositol 3-kinase/Akt and ERK pathways. Life Sci 78: 2889-2897, 2006

38. Maines MD: The heme oxygenase system: a regulator of second messenger gases. Annu Rev Pharmacol Toxicol 37: 517-554, 1997.

39. Kutty RK, Kutty G, Rodriguez IR, Chader GJ and Wiggert B: Chromosomal localization of the human heme oxygenase genes: Heme oxygenase-1 (HMOX1) maps to chromosome 22q12 and heme oxygenase-2 (HMOX2) maps to chromosome 16p13.3. Genomics 20: 513-516, 1994

40. Scarabelli TM, Knight R, Stephanou A, Townsend P, Chen-Scarabelli C, Lawrence K, Gottlieb R, Latchman D and Narula J: Clinical implications of apoptosis in ischemic myocardium. Curr Probl Cardiol 31: 181-264, 2006.

41. Saelens X, Festjens N, Vande Walle L, van Gurp M, van Loo G and Vandenabeele P: Toxic proteins released from mitochondria in cell death. Oncogene 23: 2861-2874, 2004

42. Murphy KM, Streips UN and Lock RB: Bax membrane insertion during Fas(CD95)-induced apoptosis precedes cytochrome c release and is inhibited by Bcl-2. Oncogene 18: 5991-5999, 1999.

43. Salvesen GS; Caspases and apoptosis. Essays Biochem 38: 9-19, 2002.

44. Kim KC, Kang KA, Zhang R, Piao MJ, Kim GY, Kang MY, Lee SJ, Lee NH, Surh YJ and Hyun JW: Upregulation of Nrf2-mediated heme oxygenase-1 expression by eckol, a phlorotannin compound, through activation of Erk and PI3K/Akt. Int J Biochem Cell Biol 42: 297-305, 2010.

45. Minelli A, Conte C, Grottelli S, Bellezza I, Emiliani C and Bolaños JP: Cyclo(His-Pro) upregulates heme oxygenase 1 via activation of Nrf2-ARE signalling. J Neurochem 111: 956-966, 2009.

46. Lee IS, Lim J, Gal J, Kang JC, Kim HJ, Kang BY and Choi HJ: Anti-inflammatory activity of xanthohumol involves heme oxygenase-1 induction via NRF2-ARE signaling in microglial BV2 cells. Neurochem Int 58: 153-160, 2011.

47. Ungvari Z, Bagi Z, Feher A, Recchia FA, Sonntag WE, Pearson K, de Cabo R and Csiszar A: Resveratrol confers endothelial protection via activation of the antioxidant transcription factor Nrf2. Am J Physiol Heart Circ Physiol 299: H18-H24, 2010.

48. Mann GE, Niehueser-Saran J, Watson A, Gao L, Ishii T, de Winter $P$ and Siow RC: Nrf2/ARE regulated antioxidant gene expression in endothelial and smooth muscle cells in oxidative stress: implications for atherosclerosis and preeclampsia. Sheng Li Xue Bao 59: 117-127, 2007.

49. Lee S, Park Y, Zuidema MY, Hannink M and Zhang C: Effects of interventions on oxidative stress and inflammation of cardiovascular diseases. World J Cardiol 3: 18-24, 2011.

50. Imming P, Sinning C and Meyer A: Drugs, their targets and the nature and number of drug targets. Nat Rev Drug Discov 5 : $821-834,2006$ 